\title{
Generalized Spectral Characterization of Mixed Graphs*
}

\author{
Wei Wang Lihong Qiu \\ School of Mathematics and Statistics \\ Xi'an Jiaotong University \\ Xi'an, China \\ wang_weiw@163.com, qiulihong2011@126.com \\ Jianguo Qian \\ School of Mathematical Sciences \\ Xiamen University \\ Xiamen, China \\ jgqian@xmu.edu.cn \\ Wei Wang ${ }^{\dagger}$ \\ School of Mathematics, Physics and Finance \\ Anhui Polytechnic University \\ Wuhu, China \\ wangwei.math@gmail.com
}

Submitted: May 15, 2020; Accepted: Dec 10, 2020; Published: Dec 24, 2020

(c) The authors. Released under the CC BY-ND license (International 4.0).

\begin{abstract}
The spectral characterization of graphs is an important topic in spectral graph theory, which has been studied extensively in recent years. Unlike the undirected case, however, the spectral characterization of mixed graphs (digraphs) has received much less attention so far, which will be the main focus of this paper. A mixed graph $G$ is said to be strongly determined by its generalized Hermitian spectrum (abbreviated $S H D G S$ ), if, up to isomorphism, $G$ is the unique mixed graph that is cospectral with $G$ w.r.t. the generalized Hermitian spectrum.

Let $G$ be a self-converse mixed graph of order $n$ with Hermitian adjacency matrix $A$ and let $W=\left[e, A e, \ldots, A^{n-1} e\right]$ ( $e$ is the all-one vector). Suppose that $2^{-\lfloor n / 2\rfloor} \operatorname{det} W$ is norm-free in $\mathbb{Z}[i]$ (i.e., for any Gaussian prime $p$, the norm $N(p)=$ $p \bar{p}$ does not divide $\left.2^{-\lfloor n / 2\rfloor} \operatorname{det} W\right)$. We conjecture that every such graph is SHDGS and prove that, for any mixed graph $H$ that is cospectral with $G$ w.r.t. the generalized Hermitian spectrum, there exists a Gaussian rational unitary matrix $U$ with $U e=e$ such that $U^{*} A(G) U=A(H)$ and $(1+i) U$ is a Gaussian integral matrix. We have verified the conjecture in two extremal cases when $G$ is either an undirected

*Supported by the National Natural Science Foundation of China (Grant No.12001006, 11971376 and 11971406) and the Scientific Research Foundation of Anhui Polytechnic University (Grant

${ }^{\dagger}$ Corresponding author.
\end{abstract} No. 2019YQQ024) 
graph or a self-converse oriented graph. Moreover, as consequences of our main results, we prove that all directed paths of even order are SHDGS. Analogous results are also obtained in the setting of restrictive determination by generalized Hermitian spectrum (i.e., the spectral determination within the subset of all self-converse mixed graphs), which extends a recent result of the first author on the generalized spectral characterization of undirected graphs.

Mathematics Subject Classifications: 05C50

\section{Introduction}

Let $G$ be a simple graph with $(0,1)$-adjacency matrix $A(G)$. The spectrum of $G$, denoted by $\operatorname{Spec}(G)$, is the multiset of the eigenvalues of $A(G)$. Two graphs $G$ and $H$ are cospectral if $\operatorname{Spec}(G)=\operatorname{Spec}(H)$. Trivially, isomorphic graphs are cospectral. However, the converse is not true in general. A graph $G$ is said to be determined by its spectrum (DS for short) if any graph cospectral to $G$ is isomorphic to $G$. It is a fundamental and challenging problem to characterize which graphs are DS. Although it was conjectured that almost all graphs are DS [3], it is usually extremely difficult to prove a given graph to be DS. For basic results on spectral characterization of graphs, we refer the readers to the survey papers $[3,4]$.

In recent years, Wang and $\mathrm{Xu}[18,19]$ and Wang $[15,17]$ considered a variant of the above problem. For a graph $G$, the generalized spectrum is the ordered pair $(\operatorname{Spec}(G)$, $\operatorname{Spec}(\bar{G})$ ), where $\bar{G}$ denotes the complement of $G$. A graph $G$ is said to be determined by its generalized spectrum (DGS for short) if any graph having the same generalized spectrum as $G$ is isomorphic to $G$. Let $J$ be the all-one matrix of order $n$. For $y \in \mathbb{R}$, two graphs $G$ and $H$ are $y$-cospectral if $y J-A(G)$ and $y J-A(H)$ have the same spectrum. Moreover, we say that $G$ and $H$ are $\mathbb{R}$-cospectral if $G$ and $H$ are $y$-cospectral for any $y \in \mathbb{R}$. A classical result of Johnson and Newman [6] says that if two graphs are $y$ cospectral for two distinct values of $y$ then the same is true for all $y$. Therefore, if two graphs $G$ and $H$ are cospectral with cospectral complement, i.e., $G$ and $H$ are 0-cospectral and 1-cospectral, then they are $\mathbb{R}$-cospectral.

Let $G$ be a graph with $n$ vertices, $A=A(G)$ and $e$ be the all-one vector of dimension $n$. Let $W(G)=\left[e, A e, \ldots, A^{n-1} e\right]$ be its walk-matrix. The following simple arithmetic criterion for graphs being DGS was conjectured in [15] and finally proved in [17].

Theorem 1. [15, 17] Let $G$ be a graph with $n$ vertices. If $\frac{\operatorname{det} W(G)}{2^{\lfloor n / 2\rfloor}}$ (which is always an integer) is odd and square-free, then $G$ is $D G S$.

Similar result was established for the generalized $Q$-spectrum in [11]. Moreover, Qiu et al. [12] also gave an analogue of Theorem 1 for Eulerian graphs. We try to extend Theorem 1 from ordinary graphs to mixed graphs. A mixed graph $G$ is obtained from a simple undirected graph by orientating a subset of edges. For a mixed graph $G$, the converse of $G$, denoted by $G^{\mathrm{T}}$, is the mixed graph obtained from $G$ by reversing each directed edge in $G$. A mixed graph is said to be self-converse if $G^{\mathrm{T}}$ is isomorphic to $G$. As a trivial example, each simple undirected graph is self-converse as $G^{\mathrm{T}}=G$ in this case. 
For a mixed graph $G$, we use the symbol $u \sim v$ to denote that $u v$ is an undirected edge, and use $u \rightarrow v$ (or $v \leftarrow u$ ) to denote that $u v$ is a directed edge from $u$ to $v$. We use $\mathcal{G}_{n}$ to denote the set of all mixed graphs with vertex set $V=[n]=\{1,2, \ldots, n\}$. The subset of all self-converse mixed graphs in $\mathcal{G}_{n}$ will be denoted by $\mathcal{G}_{n}^{s c}$.

The following definition introduced independently by Liu and $\mathrm{Li}$ [7], as well as Guo and Mohar [5], is a natural generalization of adjacency matrix from ordinary graphs to mixed graphs.

Definition 2. $[7,5]$ Let $G \in \mathcal{G}_{n}$. The Hermitian adjacency matrix of $G$ is the matrix $A=\left(a_{u, v}\right) \in \mathbb{C}^{n \times n}$, where

$$
a_{u, v}= \begin{cases}1 & \text { if } u \sim v \\ i & \text { if } u \rightarrow v \\ -i & \text { if } u \leftarrow v \\ 0 & \text { otherwise }\end{cases}
$$

Note that for any mixed graph $G, A(G)$ is a Hermitian matrix, that is, $A(G)^{*}=A(G)$, where $A(G)^{*}$ denotes the conjugate transpose of $A(G)$. Therefore, all eigenvalues of $A(G)$ are real and $A(G)$ is diagonalizable. Also note that $A\left(G^{\mathrm{T}}\right)$ equals $(A(G))^{\mathrm{T}}$, the transpose of $A(G)$, and this explains why we use $G^{\mathrm{T}}$ to denote the converse of $G$. For a mixed graph $G$, the (Hermitian) spectrum of $G$, denoted by $\operatorname{Spec}(G)$, is the multiset of the eigenvalues of $A(G)$. It was observed in [5] that any mixed graph $G$ is cospectral to its converse $G^{\mathrm{T}}$ since $A\left(G^{\mathrm{T}}\right)=(A(G))^{\mathrm{T}}$. Indeed, for any $y \in \mathbb{R}, y J-A\left(G^{\mathrm{T}}\right)$ and $y J-A(G)$ have the same spectrum.

Besides the operation of reversing all directed edges, Guo and Mohar [5] found another important operation, called four-way switching, which also preserves the Hermitian spectrum. It turns out that extremely rare mixed graphs are determined by their Hermitian spectra [5, 20]. Indeed, there are 1,540,944 unlabeled mixed graphs of order 6, only 16 of them are determined by their Hermitian spectra [5, Table 1]. In [2], Mohar considered the spectral determination of classes of switching equivalent mixed graphs, rather than individual graphs. A mixed graph $G$ is determined by its Hermitian spectrum in the sense of Mohar [2] (named weak determination in [20]) if every mixed graph with the same Hermitian spectrum can be obtained from $G$ by a four-way switching, possibly followed by the reversal of all directed edges; see [1] for more results along this line.

Very recently, Wissing and van Dam [20] considered the spectral determination of individual mixed graphs, which is closer to the usual sense of spectral determination of ordinary graphs. A mixed graph is said to be strongly determined by its Hermitian spectrum (abbreviated SHDS) if it is isomorphic to each mixed graph to which it is cospectral. Although this property is extremely rare, the first infinite family of connected mixed graph that is SHDS is constructed in [20].

In this paper, we shall follow Wissing and van Dam [20] along this line of research, while our main interest is the strong spectral determination of mixed graphs, in the context of generalized spectra, where the generalized spectrum of $G$ means the ordered pair $(\operatorname{Spec}(G), \operatorname{Spec}(J-I-A(G)))$. We remark that, compared with a single kind of spectrum, 
the generalized spectrum seems to be more powerful to distinguish mixed graphs. For example, among all 15,224 self-converse mixed graphs on 6 vertices, the number of mixed graphs that are strongly determined by the generalized Hermitian spectrum is 11,591 , while this number is only 16 for the Hermitian spectrum; see Table 1 in Section 4 for more details.

The following definition is a natural generalization of the DGS problem from ordinary graphs to mixed graphs.

Definition 3. A mixed graph $G \in \mathcal{G}_{n}$ is said to be strongly determined by generalized Hermitian spectrum (SHDGS) if for any $H \in \mathcal{G}_{n}$,

$$
(\operatorname{Spec}(H), \operatorname{Spec}(J-I-A(H)))=(\operatorname{Spec}(G), \operatorname{Spec}(J-I-A(G)))
$$

implies that $H$ is isomorphic to $G$.

Remark 4. For an ordinary graph $G, J-I-A(G)$ is the adjacency matrix of the complement $\bar{G}$. Such an explanation is not available for mixed graphs.

A mixed graph that is SHDGS must be self-converse. We also consider spectral determination in the range of $\mathcal{G}_{n}^{s c}$, the subset of self-converse mixed graphs in $\mathcal{G}_{n}$.

Definition 5. A mixed graph $G \in \mathcal{G}_{n}^{s c}$ is said to be restrictively determined by generalized Hermitian spectrum (RHDGS) if for any $H \in \mathcal{G}_{n}^{s c}$,

$$
(\operatorname{Spec}(H), \operatorname{Spec}(J-I-A(H)))=(\operatorname{Spec}(G), \operatorname{Spec}(J-I-A(G)))
$$

implies that $H$ is isomorphic to $G$.

Remark 6. Any SHDGS mixed graph is self-converse and certainly RHDGS. Since $\mathcal{G}_{n}^{s c}$ contains the set of undirected graphs as a proper set, any RHDGS undirected graph is DGS. Thus for an undirected graph $G, G$ is SHDGS implies that it is RHDGS, which further implies that it is DGS.

For $G \in \mathcal{G}_{n}$, we also define $W(G)=\left[e, A e, A^{2} e, \ldots, A^{n-1} e\right]$ and call it the walk-matrix of $G$. And let $\overline{W(G)}$ denote the conjugate of $W(G)$. As $W(G)$ has complex entries, the determinant of $W(G)$ is usually not real. The following simple result illustrates an important property on $W(G)$ when $G$ is self-converse.

Theorem 7. Let $G \in \mathcal{G}_{n}^{s c}$. Then there exists a permutation matrix $P$ such that $\overline{W(G)}=$ $P^{-1} W(G)$. In particular, $\operatorname{det} W(G)$ is real or pure imaginary.

Proof. As $A\left(G^{\mathrm{T}}\right)=(A(G))^{\mathrm{T}}=\overline{A(G)}$ and $\bar{e}=e$, we have

$$
W\left(G^{\mathrm{T}}\right)=\left[e, A\left(G^{\mathrm{T}}\right) e, \ldots, A^{n-1}\left(G^{\mathrm{T}}\right) e\right]=\left[\bar{e}, \overline{A(G) e}, \ldots, \overline{A^{n-1}(G) e}\right]=\overline{W(G)} .
$$

On the other hand, as $G$ is self-converse, there exists a permutation matrix $P$ such that $A\left(G^{\mathrm{T}}\right)=P^{-1} A(G) P$. As $P e=e$, we have $A^{k}\left(G^{\mathrm{T}}\right) e=P^{-1} A^{k}(G) P e=P^{-1} A^{k}(G) e$ and hence

$$
W\left(G^{\mathrm{T}}\right)=\left[P^{-1} e, P^{-1} A(G) e, \ldots, P^{-1} A^{n-1}(G) e\right]=P^{-1} W(G) .
$$


Thus $\overline{W(G)}=P^{-1} W(G)$. Taking determinants on both sides and noting that $\operatorname{det} P^{-1}=$ \pm 1 , we obtain $\operatorname{det} \overline{W(G)}= \pm \operatorname{det} W(G)$. Therefore, $\overline{\operatorname{det} W(G)}= \pm \operatorname{det} W(G)$, which implies that $\operatorname{det} W(G)$ is real or pure imaginary.

It is known that $2^{\lfloor n / 2\rfloor}$ always divides $\operatorname{det} W(G)$ for ordinary graph $G$. We shall show that this fact can be extended to mixed graphs in the sense of Gaussian integers. As a generalization of Theorem 1, we propose the following

Conjecture 8. Let $G \in \mathcal{G}_{n}^{s c}$. If $\frac{|\operatorname{det} W(G)|}{2^{\lfloor n / 2\rfloor} \mid}$ is odd and square-free, then $G$ is RHDGS. If, in addition, each prime factor of $\frac{|\operatorname{det} W(G)|}{2^{\lfloor n / 2\rfloor}}$ is of the form $4 k+3$, then $G$ is SHDGS.

Note that the first half of this conjecture (if true) strengthens Theorem 1 . We have verified the conjecture for $n \leqslant 6$ using nauty package [9] in SageMath [13]; see Table 1 in Section 4. The main objective of this paper is to give more evidences to support Conjecture 8 .

The rest of the paper is organized as follows. In Section 2, we review some basic facts about Gaussian integers and Gaussian rational unitary matrix. In Section 3, we give some divisibility relations that will be needed later in the paper. In Section 4, we present the main result of the paper together with its proof, which strongly supports our main conjecture above. In Section 5, we verify this conjecture in two extremal cases. Conclusions and future work are given in Section 6.

\section{Gaussian rational unitary matrix and its level}

We recall some facts about Gaussian integers.

The Gaussian integers are the elements of the set $\mathbb{Z}[i]=\{a+b i: a, b \in \mathbb{Z}\}$, where $i=\sqrt{-1}$. For a Gaussian integer $z=a+b i$, the norm of $z$ is $N(z)=a^{2}+b^{2}$. Note that $N\left(z_{1} z_{2}\right)=N\left(z_{1}\right) N\left(z_{2}\right)$. The units of $\mathbb{Z}[i]$ are four powers of $i$, that is, $i,-1,-i, 1$. Two Gaussian integers $z_{1}$ and $z_{2}$ are associates if $b=u a$ for some unit $u$. It is well known that $\mathbb{Z}[i]$ is a Euclidean domain and hence a unique factorization domain. A nonzero Gaussian integer $z$ is a Gaussian prime if it is not a unit and is divisible only by units and its associates. A positive prime in $\mathbb{Z}$ is a Gaussian prime if and only if $p \equiv 3(\bmod 4)$. If $p$ is positive prime in $\mathbb{Z}$ such that $p \equiv 1(\bmod 4)$ then $p$ can be factored uniquely to a product of two conjugate Gaussian primes (up to multiplication by units and the order of the factors). For example, $5=(1+2 i)(1-2 i)=(2+i)(2-i)$. In addition, 2 is not a Gaussian prime, as $2=(1+i)(1-i)$.

We call a Gaussian integer $z$ even if $\operatorname{Re}(z)-\operatorname{Im}(z) \equiv 0(\bmod 2)$; and odd otherwise. We call $z \in \mathbb{Z}[i]$ square-free if $p^{2} \nmid z$; and norm-free if $N(p) \nmid z(N(p)=p \bar{p}$ is the norm of $p$ ), for any Gaussian prime $p$. Note that the concept of norm-free over $\mathbb{Z}[i]$ is a natural generalization of that of square-free over $\mathbb{Z}$. In particular, 2 is neither square-free nor norm-free. All ordinary odd primes are square-free, but only primes of the form $4 k+3$ are norm-free. We write it down as the following fact, which will be used to give a more compact and natural formulation for Conjecture 8. 
Fact 9. Let $z$ be a real or pure imaginary integer. Then

(1) $z$ is square-free in $\mathbb{Z}[i]$ if and only if $|z|$ is odd and square-free in the ordinary sense.

(2) $z$ is norm-free in $\mathbb{Z}[i]$ if and only if $|z|$ is odd, square-free in the ordinary sense and each ordinary prime factor of $|z|$ is of the form $4 k+3$.

For a Gaussian prime $p=a+b i$, the quotient ring $\mathbb{Z}[i] /(p)=G F(N(p))$, where $G F(N(p))$ is the Galois field of order $N(p)=a^{2}+b^{2}$. As a simple example, $1+i$ is a Gaussian prime and $\mathbb{Z}[i] /(1+i)$ is the binary field $G F(2)$.

A Gaussian rational number is a complex number whose real part and imaginary part are rational. A unitary matrix is a matrix $U \in \mathbb{C}^{n \times n}$ satisfying $U^{*} U=I$. A matrix $U \in \mathbb{C}^{n \times n}$ is said to be Gaussian rational unitary if $U$ is unitary and each entry of $U$ is Gaussian rational. The following result is a natural generalization of a result for adjacency matrix of an undirected graph obtained in $[6,19]$. The proof is omitted here since the previous proof is also valid by some slight and evident modifications.

Theorem 10. Let $G \in \mathcal{G}_{n}$. There exists $H$ such that $G$ and $H$ are cospectral with respect to the generalized Hermitian spectrum if and only if there exists a unitary matrix $U$ satisfying

$$
U^{*} A(G) U=A(H), U e=e .
$$

Moreover, if $\operatorname{det} W(G) \neq 0$ then $U=W(G) W^{-1}(H)$ and hence is unique and Gaussian rational.

Let $G, H \in \mathcal{G}_{n}$. Define

$$
\mathscr{U}_{G}(H)=\left\{U \text { is Gaussian rational unitary: } U^{*} A(G) U=A(H) \text { and } U e=e\right\},
$$

and $\mathscr{U}_{G}=\cup \mathscr{U}_{G}(H)$, where the union is taken over all $H \in \mathcal{G}_{n}$. Similarly, we define $\mathscr{U}_{G}^{s c}=\cup \mathscr{U}_{G}(H)$, where $G \in \mathcal{G}_{n}^{s c}$ and the union is taken over all $H \in \mathcal{G}_{n}^{s c}$.

Under the assumption that $\operatorname{det} W(G) \neq 0$, the structure of $\mathscr{U}_{G}(H)$ is simple. It is either a singleton or an empty set depending on whether (2) holds or not. Furthermore, if (2) holds, then $\mathscr{U}_{G}(H)=\left\{W(G) W^{-1}(H)\right\}$. In addition, if $G$ and $H$ are isomorphic, i.e., there exists a permutation matrix $P$ with $P^{*} A(G) P=A(H)$, then $\mathscr{U}_{G}(H)=\{P\}$ as $P$ is clearly Gaussian rational unitary and $P e=e$. On the other hand, if (2) holds but $H$ is not isomorphic to $G$, then the unique element in $\mathscr{U}_{G}(H)$ is not a permutation matrix.

Therefore, if $G$ is SHDGS, then either $\mathscr{U}_{G}(H)=\emptyset$ or $\mathscr{U}_{G}(H)$ consists of a single permutation matrix. Thus, $\mathscr{U}_{G}$ contains only permutation matrices. If $G$ is not SHDGS, then there exists an $H$ such that (2) holds but $H$ is not isomorphic to $G$. For such an $H$, the matrix in $\mathscr{U}_{G}(H)$ is not a permutation matrix and hence $\mathscr{U}_{G}$ contains matrices other than permutation matrices. Similar analysis is also valid for restrictive determination. We summarize this as the following theorem, which was observed in [19] for ordinary graphs.

Theorem 11. Let $G \in \mathcal{G}_{n}^{s c}$ such that $\operatorname{det} W(G) \neq 0$. Then $G$ is $S H D G S$ (resp. RHDGS) if and only if $\mathscr{U}_{G}$ (resp. $\mathscr{U}_{G}^{s c}$ ) contains only permutation matrices. 
Let

$$
\Gamma=\{z \in \mathbb{Z}[i]: \operatorname{Re}(z)>0, \operatorname{Im}(z) \geqslant 0\} .
$$

It is easy to see that $(\Gamma, i \cdot \Gamma,-\Gamma,-i \cdot \Gamma)$ is a partition of $\mathbb{Z}[i] \backslash\{0\}$, where $i \cdot \Gamma=\{i z: z \in \Gamma\}$. Thus, for any nonzero Gaussian integer $z$, exactly one of its four associates lies in $\Gamma$.

Definition 12. Let $U$ be a Gaussian rational unitary matrix. The level of $U$ is the Gaussian integer $\ell=\ell(U) \in \Gamma$ such that $\ell U$ is a Gaussian integral matrix and $N(\ell)$ is minimal.

The assumption $\ell(U) \in \Gamma$ makes $\ell(U)$ unique and hence well-defined. We will make similar convention on least common multiple (LCM) and greatest common divisor (GCD) on Gaussian integers. We note that $\ell$ is the lcm of all denominators (in the form of reduced fraction) of all entries in $U$. In particular, if $g U$ is a Gaussian integral matrix then $\ell \mid g$. Clearly, a Gaussian rational unitary matrix $U$ with $U e=e$ is a permutation matrix if and only if $\ell(U)=1$. The following observation illustrates the special property of $\mathscr{U}_{G}^{s c}$, which $\mathscr{U}_{G}$ does not necessarily have.

Theorem 13. Let $G \in \mathcal{G}_{n}^{s c}$ such that $\operatorname{det} W(G) \neq 0$. For any $U \in \mathscr{U}_{G}^{s c}, \ell(U)$ and $\overline{\ell(U)}$ are associates, that $i s, \ell(U)=a$ or $\ell(U)=a(1+i)$ for some positive integer a.

Proof. Let $H \in \mathcal{G}_{n}^{s c}$ such that $U \in \mathscr{U}_{G}(H)$. Thus, $U=W(G) W^{-1}(H)$. Since both $G$ and $H$ are self-converse, it follows from Theorem 7 that there exist two permutation matrices $P$ and $Q$ such that $\overline{W(G)}=P^{-1} W(G)$ and $\overline{W(H)}=Q^{-1} W(H)$. Therefore,

$$
\bar{U}=\overline{W(G) W^{-1}(H)}=P^{-1} W(G) W(H)^{-1} Q=P^{-1} U Q .
$$

Thus, $\overline{\ell(U)} U=\overline{\ell(U) \bar{U}}=P^{-1} \overline{\ell(U) U} Q$ and hence $\overline{\ell(U)} U$ is Gaussian integral. Moreover, due to the minimality of $\ell(U)$, we have $\ell(U) \mid \overline{\ell(U)}$. Taking conjugate we have $\overline{\ell(U)} \mid \ell(U)$ and hence $\ell(U)$ and $\overline{\ell(U)}$ are associates. Since $\ell(U) \in \Gamma$, we find that the amplitude of $\ell(U)$ is either 0 or $\frac{\pi}{4}$. That is, $\ell(U)=a$ or $\ell(U)=a(1+i)$ for some positive integer $a$.

Example 14. Let $G$ and $H$ be two mixed graphs as shown in Fig. 1. Note that $G$ is self-converse but $H$ is not. An exhaustive search indicates that $H$ and $H^{\mathrm{T}}$ are the only two graphs (up to isomorphism) which are $\mathbb{R}$-cospectral but not isomorphic to $G$. Thus, $G$ is RHDGS but not SHDGS. In other words, $\mathscr{U}_{G}^{s c}$ contains only permutation matrices while $\mathscr{U}_{G}$ contains matrices other than permutation matrices. In particular, $W(G) W^{-1}(H) \in \mathscr{U}_{G}$ and a direct calculation shows that

$$
W(G) W^{-1}(H)=\frac{1}{4+i}\left(\begin{array}{ccccc}
2+i & 1-i & 2+2 i & -1-i & 0 \\
-1+i & -i & 2 & 3+i & 0 \\
2-2 i & 2 i & i & 2 & 0 \\
1+i & 3+i & -2 i & i & 0 \\
0 & 0 & 0 & 0 & 4+i
\end{array}\right) .
$$

Clearly $W(G) W^{-1}(H)$ has level $4+i$, which is not an associate of its conjugate $4-i$. 

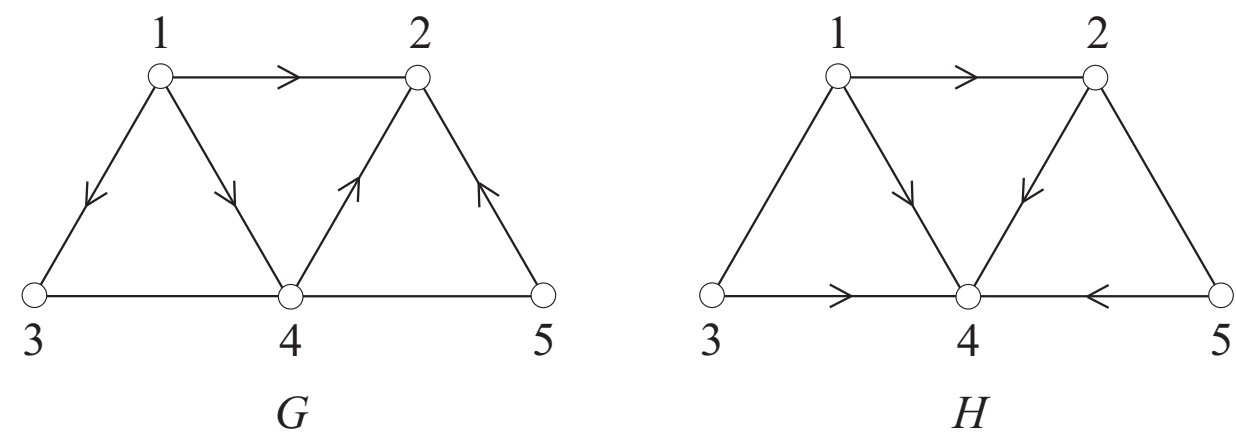

Figure 1: Two $\mathbb{R}$-cospectral but not isomorphic graphs

\section{Some divisibility relations}

The following lemma was first established for ordinary graphs in [16]. The original proof can be easily extended to mixed graphs. For simplicity, we shall write $A=A(G)$ and $W=W(G)$ in the rest of the paper.

Lemma 15. Let $G \in \mathcal{G}_{n}$. Then for any positive integer $k, e^{*} A^{k} e \equiv 0(\bmod 2)$.

Proof. Denote the $(i, j)$-entry of $A^{k}$ as $a_{i, j}^{(k)}$. Note that $A^{k}$ is Hermitian as $A$ is Hermitian. Thus, we have

$$
e^{*} A^{k} e=\operatorname{Tr}\left(A^{k}\right)+\sum_{i<j}\left(a_{i, j}^{(k)}+\overline{a_{i, j}^{(k)}}\right)=\operatorname{Tr}\left(A^{k}\right)+\sum_{i<j} 2 \operatorname{Re}\left(a_{i, j}^{(k)}\right) \equiv \operatorname{Tr}\left(A^{k}\right) \quad(\bmod 2) .
$$

On the other hand, as all diagonal entries of $A$ are zero, we have

$$
\begin{aligned}
\operatorname{Tr}\left(A A^{k-1}\right) & =\sum_{i \neq j} a_{i, j}^{(1)} a_{j, i}^{(k-1)} \\
& =\sum_{i<j}\left(a_{i, j}^{(1)} a_{j, i}^{(k-1)}+\overline{a_{i, j}^{(1)} a_{j, i}^{(k-1)}}\right) \\
& =\sum_{i<j} 2 \operatorname{Re}\left(a_{i, j}^{(1)} a_{j, i}^{(k-1)}\right) \\
& \equiv 0(\bmod 2) .
\end{aligned}
$$

Therefore, $e^{*} A^{k}(G) e \equiv 0(\bmod 2)$. This proves the lemma.

Let $p$ be a Gaussian prime and $M$ be a Gaussian integral matrix, we use $\operatorname{rank}_{p}(M)$ to denote the rank of $M$ over the field $\mathbb{Z}[i] /(p)$. Note that $\operatorname{rank}_{\bar{p}}(\bar{M})=\operatorname{rank}_{p}(M)$ always holds. In addition, if $p$ and $\bar{p}$ are associates, then we have $\operatorname{rank}_{\bar{p}}(M)=\operatorname{rank}_{p}(M)$. In particular, $\operatorname{rank}_{1+i}(M)=\operatorname{rank}_{1-i}(M)$.

Corollary 16. Let $G \in \mathcal{G}_{n}$. Then the followings hold.

(1) $2^{\left\lfloor\frac{n}{2}\right\rfloor} \mid \operatorname{det} W$, and

(2) $\operatorname{rank}_{1+i} W \leqslant\left\lceil\frac{n}{2}\right\rceil$. 
Proof. Let $M=W^{*} W$. Let $m_{i, j}$ denote the $(i, j)$-entry of $M$. Then $m_{i, j}=e^{*} A^{i+j-2} e$. Note that $m_{1,1}=n$. Thus, by Lemma $15, m_{i, j} \equiv 0(\bmod 2)$ unless $(i, j)=(1,1)$ and $n$ is odd. Therefore, $2^{n} \mid \operatorname{det} M$ when $n$ is even, and $2^{n-1} \mid \operatorname{det} M$ when $n$ is odd. In other words,

$$
2^{2\left\lfloor\frac{n}{2}\right\rfloor} \mid \operatorname{det} M .
$$

As 2 and $(1+i)^{2}$ are associates and $\operatorname{det} M=\operatorname{det} W^{*} \operatorname{det} W=\overline{\operatorname{det} W} \operatorname{det} W$, we can rewrite (9) as

$$
(1+i)^{4\left\lfloor\frac{n}{2}\right\rfloor} \mid \overline{\operatorname{det} W} \operatorname{det} W .
$$

As $\overline{1+i}$ and $1+i$ are associates, from (10), we have

$$
(1+i)^{4\left\lfloor\frac{n}{2}\right\rfloor} \mid(\operatorname{det} W)^{2}
$$

and hence $(1+i)^{2\left\lfloor\frac{n}{2}\right\rfloor} \mid \operatorname{det} W$, i.e., $2^{\left\lfloor\frac{n}{2}\right\rfloor} \mid \operatorname{det} W$. This proves (1).

Note that $m_{i, j} \equiv 0(\bmod 1+i)$ unless $(i, j)=(1,1)$ and $n$ is odd. We have

$$
\operatorname{rank}_{1+i} M= \begin{cases}0 & \text { if } n \text { is even } \\ 1 & \text { if } n \text { is odd }\end{cases}
$$

Using the familiar inequality that $\operatorname{rank} B+\operatorname{rank} C \leqslant n+\operatorname{rank} B C$ for any matrix of order $n$, we have

$$
\operatorname{rank}_{1+i} W^{*}+\operatorname{rank}_{1+i} W \leqslant n+\operatorname{rank}_{1+i} M .
$$

Note that $\operatorname{rank}_{1+i} W^{*}=\operatorname{rank}_{1+i} W$, which combining with (13) implies

$$
\operatorname{rank}_{1+i} W \leqslant\left\lfloor\frac{n+\operatorname{rank}_{1+i} M}{2}\right\rfloor .
$$

Clearly, using (12), the right term in (14) can be reduced to $\left\lceil\frac{n}{2}\right\rceil$. This proves (2).

Lemma 17. Let $G \in \mathcal{G}_{n}$ and $r=\operatorname{rank}_{1+i} W$. Then the first $r$ columns of $W$ are linearly independent over $\mathbb{Z}[i] /(1+i)$.

Proof. Suppose to the contrary that $e, A e, \ldots, A^{r-1} e$ are linearly dependent. Then there exists an integer $m$ such that $m \leqslant r-1$ and

$$
A^{m} e \in \operatorname{Span}\left\{e, A e, \ldots, A^{m-1} e\right\} .
$$

Using (15) twice, we have

$$
A^{m+1} e \in \operatorname{Span}\left\{A e, A^{2} e, \ldots, A^{m} e\right\} \subseteq \operatorname{Span}\left\{e, A e, \ldots, A^{m-1} e\right\} .
$$

Similarly, for any $m^{\prime}>m$, we always have $A^{m^{\prime}} e \in \operatorname{Span}\left\{e, A e, \ldots, A^{m-1} e\right\}$. Thus, $\operatorname{rank}_{1+i} W \leqslant m<r=\operatorname{rank}_{1+i} W$. This contradiction completes the proof of this lemma. 
The following result gives a basic relation between $\operatorname{rank}_{1+i} W$ and $\operatorname{det} W$. We note that the real counterpart is easy to obtain using Smith Normal Form and the fact that 2 is a prime in $\mathbb{Z}$. Unfortunately, similar argument is not valid since 2 is factorable in $\mathbb{Z}[i]$. Some new techniques have to be used to overcome this difficulty.

Lemma 18. Let $G \in \mathcal{G}_{n}^{s c}$ and $r=\operatorname{rank}_{1+i} W$. Then we have

$$
2^{n-r} \mid \operatorname{det} W
$$

Proof. By Corollary 16, we have $r \leqslant\left\lceil\frac{n}{2}\right\rceil$ and $2^{\left\lfloor\frac{n}{2}\right\rfloor} \mid \operatorname{det} W$. If $r=\left\lceil\frac{n}{2}\right\rceil$ then $n-r=\left\lfloor\frac{n}{2}\right\rfloor$ and hence (17) holds. Thus, it suffices to consider the case that $r<\left\lceil\frac{n}{2}\right\rceil$.

By Lemma 17, $A^{r} e$ can be expressed as a linear combination of $A^{r-1} e, A^{r-2} e, \ldots, e$ over $\mathbb{Z}[i] /(1+i)$. Let $\left(c_{1}, c_{2}, \ldots, c_{r}\right)$ be a $0-1$ vector such that $A^{r} e \equiv c_{1} A^{r-1} e+c_{2} A^{r-2} e+\cdots+c_{r} e$ $(\bmod 1+i)$. Let $B=A^{r}-c_{1} A^{r-1}-c_{2} A^{r-2}-\cdots-c_{r} I$. Note that $B$ is Hermitian.

Claim 1: $e^{*} B A^{k} B e \equiv 0(\bmod 4)$ for any $k \geqslant 0$.

As $B e \equiv 0(\bmod 1+i), \frac{B e}{1+i}$ is a Gaussian integral vector. Write $g=\frac{B e}{1+i}$. Now we have $e^{*} B A^{k} B e=2 g^{*} A^{k} g$. Thus, it suffices to show that $g^{*} A^{k} g \equiv 0(\bmod 2)$. We consider two cases:

Case 1: $k$ is odd, say, $k=2 s+1$.

Write $A^{s} g=h=\left(h_{1}, h_{2}, \ldots, h_{n}\right)$. Note that $A$ is Hermitian with vanishing diagonal entries. We have

$$
\begin{aligned}
g^{*} A^{k} g & =h^{*} A h \\
& =\sum_{s \neq t} \overline{h_{s}} a_{s, t} h_{t} \\
& =\sum_{s<t}\left(\overline{h_{s}} a_{s, t} h_{t}+\overline{h_{t}} a_{t, s} h_{s}\right) \\
& =\sum_{s<t}\left(\overline{h_{s}} a_{s, t} h_{t}+\overline{\overline{h_{s}}} a_{s, t} h_{t}\right) \\
& \equiv 0(\bmod 2) .
\end{aligned}
$$

Case 2: $k$ is even, say, $k=2 s$.

As $g^{*} A^{k} g$ is real, we only need to prove $g^{*} A^{k} g \equiv 0(\bmod 1+i)$. Note that for any Gaussian integer $c, \bar{c} c \equiv c(\bmod 1+i)$. Thus,

$$
g^{*} A^{k} g=\left(A^{s} g\right)^{*}\left(A^{s} g\right) \equiv e^{*}\left(A^{s} g\right) \quad(\bmod 1+i) .
$$

As $g=\frac{B e}{1+i}$, we are done if

$$
e^{*} A^{s} B e \equiv 0 \quad(\bmod 2) .
$$

By Lemma $15, e^{*} A^{j} e \equiv 0(\bmod 2)$ for any $j \geqslant 1$. Thus, if $s \geqslant 1$ then (19) holds as $B$ is a linear combination of $A^{0}, A^{1}, \ldots, A^{r-1}$. If $s=0$ then $e^{*} A^{s} B e=e^{*} B e \equiv 0(\bmod 1+i)$ as $B e \equiv 0(\bmod 1+i)$. Note that $e^{*} B e$ is real. This implies that $e^{*} B e \equiv 0(\bmod 2)$ and hence (19) always holds.

Combining Case 1 and Case 2, Claim 1 follows. 
Define $\hat{W}=\left(e, A e, \ldots, A^{r-1} e, B e, B A e, \ldots, B A^{n-r-1}\right)$. Clearly,

$$
\operatorname{det} \hat{W}=\operatorname{det} W \text {. }
$$

Write $\hat{W}=\left(W_{1}, W_{2}\right)$, where,

$$
W_{1}=\left(e, A e, \ldots, A^{r-1} e\right) \text { and } W_{2}=B\left(e, A e, \ldots, A^{n-r-1} e\right) .
$$

Now we have

$$
\hat{W}^{*} \hat{W}=\left(\hat{W}^{*} W_{1}, \hat{W}^{*} W_{2}\right)=\left(\begin{array}{ll}
W_{1}^{*} W_{1} & W_{1}^{*} W_{2} \\
W_{2}^{*} W_{1} & W_{2}^{*} W_{2}
\end{array}\right) .
$$

Write $W_{1}^{*} W_{2}=\left(f^{(1)}, f^{(2)}, \ldots, f^{(n-r)}\right)$. Note that $n-r \geqslant r+1$ as $r<\left\lceil\frac{n}{2}\right\rceil$.

Claim 2: $f^{(j)}-c_{1} f^{(j-1)}-c_{2} f^{(j-2)}-\cdots-c_{r} f^{(j-r)} \equiv 0(\bmod 4)$ for $j=r+1, r+2, \ldots, n-r$.

Let $f_{t}^{(s)}$ denote the $t$-th entry of $f^{(s)}$ for $s \in\{1,2, \ldots, n-r\}$ and $t \in\{1,2, \ldots, r\}$. Note that $A$ and $B$ are commutative. one can easily finds that $f_{t}^{(s)}=e^{*} B A^{t+s-2} e$. Thus,

$$
\begin{aligned}
& f_{t}^{(j)}-c_{1} f_{t}^{(j-1)}-c_{2} f_{t}^{(j-2)}-\cdots-c_{r} f_{t}^{(j-r)} \\
= & e^{*} B\left(A^{t+j-2}-c_{1} A^{t+j-3}-\cdots-c_{r} A^{t+j-r-2}\right) e \\
= & e^{*} B A^{t+j-r-2}\left(A^{r}-c_{1} A^{r-1}-\cdots-c_{r} A^{0}\right) e \\
= & e^{*} B A^{t+j-r-2} B e \\
\equiv & 0(\bmod 4),
\end{aligned}
$$

where the last congruence follows from Claim 1. This proves Claim 2.

Let $F^{(j)}$ denote the $j$-th column of $\hat{W}^{*} W_{2}$ for $j=r+1, r+2, \ldots, n-r$, that is,

$$
F^{(j)}=\left(\begin{array}{c}
f^{(j)} \\
q^{(j)}
\end{array}\right)
$$

where $q^{(j)}$ denotes the $j$-th column of $W_{2}^{*} W_{2}$. Define $\hat{F}^{(j)}=F^{(j)}-c_{1} F^{(j-1)}-c_{2} F^{(j-2)}-$ $\cdots-c_{r} F^{(j-r)}$. Note that by Claim 1 , each entry of $W_{2}^{*} W_{2}$ is a multiple of 4 . Combining this fact with Claim 2, we find that $\hat{F}^{(j)} \equiv 0(\bmod 4)$. Let

$$
M=\left(\hat{W}^{*} W_{1}, F^{(1)}, F^{(2)}, \ldots, F^{(r)}, \hat{F}^{(r+1)}, \hat{F}^{(r+2)}, \ldots, \hat{F}^{(n-r)}\right) .
$$

As $\hat{W}^{*} \hat{W}=\left(\hat{W}^{*} W_{1}, \hat{W}^{*} W_{2}\right)=\left(\hat{W}^{*} W_{1}, F^{(1)}, F^{(2)}, \ldots, F^{(n-r)}\right)$, we can easily find that $\operatorname{det} \hat{W}^{*} \hat{W}=\operatorname{det} M$. Together with (20), we have

$$
\operatorname{det} M=\overline{\operatorname{det} W} \operatorname{det} W \text {. }
$$

By Lemma 15, all entries of the real matrix $\left(\hat{W}^{*} W_{1}, F^{(1)}, F^{(2)}, \ldots, F^{(r)}\right)$ are even, except the upper left corner when $n$ is odd. As $\hat{F}^{(j)} \equiv 0(\bmod 4)$ for each $j \in\{r+1, r+$ $2, \ldots, n-r\}$, we find that $2^{2 r} 4^{n-2 r} \mid \operatorname{det} M$ when $n$ is even and $2^{2 r-1} 4^{n-2 r} \mid \operatorname{det} M$ when $n$ is odd. If $n$ is even then $(1+i)^{4 n-4 r} \mid \overline{\operatorname{det} W} \operatorname{det} W$ and hence $(1+i)^{2 n-2 r} \mid \operatorname{det} W$, i.e., $2^{n-r} \mid \operatorname{det} W$. If $n$ is odd then $(1+i)^{4 n-4 r-2} \mid \overline{\operatorname{det} W} \operatorname{det} W$ and hence $(1+i)^{2 n-2 r-1} \mid \operatorname{det} W$, i.e., $2^{n-r-1}(1+i) \mid \operatorname{det} W$. Fortunately, since $G$ is self-converse, $\operatorname{det} W$ is real or pure imaginary by Theorem 7 . Now $2^{n-r-1}(1+i) \mid \operatorname{det} W$ is equivalent to $2^{n-r} \mid \operatorname{det} W$. This completes the proof of this lemma. 
Corollary 19. Let $G \in \mathcal{G}_{n}^{s c}$. If $\frac{\operatorname{det} W}{2^{\left\lfloor\frac{n}{2}\right\rfloor}}$ is odd, then $\operatorname{rank}_{1+i} W=\left\lceil\frac{n}{2}\right\rceil$.

Proof. Let $r=\operatorname{rank}_{1+i} W$. By Corollary 16, $r \leqslant\left\lceil\frac{n}{2}\right\rceil$. Suppose to the contrary that $r<\left\lceil\frac{n}{2}\right\rceil$. Then $n-r \geqslant\left\lfloor\frac{n}{2}\right\rfloor+1$. By Lemma 18, we have $2^{n-r} \mid \operatorname{det} W$ and hence $2^{\left\lfloor\frac{n}{2}\right\rfloor+1} \mid \operatorname{det} W$. This is a contradiction.

An $n \times n$ Gaussian integral matrix $U$ is called unimodular if $\operatorname{det} U$ is a unit in $\mathbb{Z}[i]$, i.e., $\operatorname{det} U=i^{k}$ for some $k \in[4]$. The following result is well known.

Lemma 20. For every $n \times n$ Gaussian integral matrix $M$ with full rank, there exist unimodular matrices $V_{1}$ and $V_{2}$ such that $M=V_{1} S V_{2}$, where $S=\operatorname{diag}\left(d_{1}, d_{2}, \ldots, d_{n}\right)$ is a Gaussian integral diagonal matrix with $d_{i}$ being the $i$-th entry in the diagonal and $d_{i} \mid d_{i+1}$ for $i=1,2, \ldots, n-1$.

For a Gaussian integral matrix $M$, the above $S$ is called the Smith Normal Form (SNF for short) of $M$ and $d_{i}$ is called the $i$-th invariant factor of the matrix. The $i$-th invariant factor is unique up to multiplication by units. For clarity, we always assume $d_{i} \in \Gamma \cup\{0\}$. The following lemma appeared in [15] for ordinary integral matrix. The proof given in [15] is of course valid for Gaussian integral matrix. We include the short proof here for the convenience of readers.

Lemma 21. [15] Let $p$ be a Gaussian prime and $M$ be an $n \times n$ Gaussian integral matrix. Then $M z \equiv 0\left(\bmod p^{2}\right)$ has a solution $z \not \equiv 0(\bmod p)$ if and only if $p^{2} \mid d_{n}$.

Proof. Let $V_{1}$ and $V_{2}$ be unimodular matrices such that $M=V_{1} \operatorname{diag}\left(d_{1}, d_{2}, \ldots, d_{n}\right) V_{2}$. The equation $M z \equiv 0\left(\bmod p^{2}\right)$ is equivalent to $\operatorname{diag}\left(d_{1}, d_{2}, \ldots, d_{n}\right) V_{2} z \equiv 0\left(\bmod p^{2}\right)$. Let $y=V_{2} z$ and consider $\operatorname{diag}\left(d_{1}, d_{2}, \ldots, d_{n}\right) y \equiv 0\left(\bmod p^{2}\right)$. If $p^{2} \mid d_{n}$, let $y=$ $(0,0, \ldots, 1)^{\mathrm{T}}$, then $z=V_{2}^{-1} y \not \equiv 0(\bmod p)$ is a required solution to the original congruence equation. On the other hand, if $p^{2} \nmid d_{n}$, then the fact that $p$ is Gaussian prime implies that $\operatorname{diag}\left(d_{1}, d_{2}, \ldots, d_{n}\right) y \equiv 0\left(\bmod p^{2}\right)$ has no solution $y$ with $y \not \equiv 0(\bmod p)$, i.e., $\operatorname{diag}\left(d_{1}, d_{2}, \ldots, d_{n}\right) V_{2} z \equiv 0\left(\bmod p^{2}\right)$ has no solution of $z$ with $z \not \equiv 0(\bmod p)$.

Lemma 22. Let $G \in \mathcal{G}_{n}^{s c}$. Then for each $i \in\{1,2, \ldots, n\}, d_{i}$ and $\overline{d_{i}}$ are associates, where $d_{i}$ is the $i$-th invariant factor of $W$. In particular, $W$ and $W^{*}$ have the same $\mathrm{SNF}$.

Proof. By Lemma 20, there exist unimodular matrices $S$ and $T$ such that $W=S \Lambda T$, where $\Lambda=\operatorname{diag}\left(d_{1}, d_{2}, \ldots, d_{n}\right)$ is the SNF of $W$. Thus, $\bar{W}=\bar{S} \bar{\Lambda} \bar{T}$. On the other hand, by Theorem 7 , there exists a permutation matrix $P$ such that $\bar{W}=P^{-1} W$. Thus, $\bar{W}=$ $\left(P^{-1} S\right) \Lambda T$. Therefore, $\overline{d_{i}}$ and $d_{i}$ are associates. Finally, as $W^{*}=(\bar{W})^{\mathrm{T}}=T^{\mathrm{T}} \Lambda\left(S^{\mathrm{T}} P\right), W$ and $W^{*}$ have the same SNF.

Lemma 23. Let $G \in \mathcal{G}_{n}^{s c}$ such that $\operatorname{det} W \neq 0$. Then for any $U \in \mathscr{U}_{G}$ we have $\ell(U) \mid d_{n}$, where $d_{n}$ is the $n$-th invariant factor of $W$.

Proof. Let $H \in \mathcal{G}_{n}$ such that $\mathscr{U}_{G}=\{U\}$. By Theorem 10, we have $U=W(G) W^{-1}(H)$. As $U^{*} U=I, U=\left(U^{*}\right)^{-1}=\left(W^{*}(G)\right)^{-1} W^{*}(H)$. By Lemma 20, there exist unimodular 
matrices $S$ and $T$ such that $W^{*}(G)=S \Lambda T$, where $\Lambda=\operatorname{diag}\left(d_{1}, d_{2}, \ldots, d_{n}\right)$ is the SNF of $W^{*}(G)$ (or $W(G)$ equivalently due to Lemma 22 ). Now we can write

$$
U=T^{-1} \operatorname{diag}\left(d_{1}^{-1}, d_{2}^{-1}, \ldots, d_{n}^{-1}\right) S^{-1} W^{*}(H) .
$$

As $T^{-1}, S^{-1}, d_{n} \operatorname{diag}\left(d_{1}^{-1}, d_{2}^{-1}, \ldots, d_{n}^{-1}\right)$ and $W^{*}(H)$ are Gaussian integral, we see that $d_{n} U$ is Gaussian integral, and hence $\ell(U) \mid d_{n}$.

\section{Main results}

In this section, we shall present the main results of the paper. Before doing so, for convenience, we restate Conjecture 8 in an equivalent form using Fact 9.

Conjecture 24. Let $G \in \mathcal{G}_{n}^{s c}$. If $\frac{\operatorname{det} W}{2^{\lfloor n / 2\rfloor}}$ is norm-free (resp. square-free) in $\mathbb{Z}[i]$, then $\ell(U)=1$ for any $U \in \mathscr{U}_{G}$ (resp. $U \in \mathscr{U}_{G}^{s c}$ ).

We have verified the above conjecture for mixed graphs of order at most 6 . The exhaustive search results are summarized in Table1, where we use $\left[\mathcal{G}_{n}^{s c}\right]$ to denote the isomorphic class of $\mathcal{G}_{n}^{s c}$, i.e., the set of unlabeled self-converse mixed graph of order $n$. It can be seen that the fractions of graphs that are SHDGS (resp. RHDGS) among all self-converse graphs of order $n$ are comparatively high, e.g, as $n=6$, the ratio of SHDGS (resp. RHDGS)) graphs is about $76 \%$ (resp. 83\%). Moreover, the ratio of self-converse mixed graphs satisfying the conditions of Conjecture 24 is not too small. For example, when $n=6$, about $4 \%$ (resp. 6.5\%) of SHDGS (resp. RHDGS)) graphs satisfy the conditions of Conjecture 24.

Table 1: Spectral determination of small mixed graphs

\begin{tabular}{|c|c|c|c|c|c|}
\hline Order $n$ of graphs & 2 & 3 & 4 & 5 & 6 \\
\hline converse mixed graphs & 3 & 10 & 70 & 708 & 15224 \\
\hline \# & 1 & 2 & 3 & 5 & 16 \\
\hline$\# C$ & 3 & 10 & 61 & 530 & 11591 \\
\hline$\# G \in\left[\mathcal{G}_{n}^{s c}\right]$ such t & 1 & 1 & 6 & 39 & 464 \\
\hline$\# G \in\left[\mathcal{G}_{n}^{s c}\right]$ restrictively determined by generalized H-sp & 3 & 10 & 64 & 603 & 12662 \\
\hline$\# G \in\left[\mathcal{G}_{n}^{s c}\right]$ such that $\frac{\operatorname{det} W}{2^{\lfloor n / 2\rfloor}}$ is square-free & 1 & 1 & 6 & 54 & 826 \\
\hline
\end{tabular}

The following theorem lies at the heart of this paper.

Theorem 25. Let $G \in \mathcal{G}_{n}^{s c}$. If $\frac{\operatorname{det} W}{2^{\lfloor n / 2\rfloor}}$ is square-free in $\mathbb{Z}[i]$, then for any $U \in \mathscr{U}_{G}$, $\operatorname{gcd}(\ell(U), \overline{\ell(U)}) \in\{1,1+i\}$.

The proof of the above theorem will be given at the end of this section. As a strong support to Conjecture 24, the main results of this paper are summarized in the following two theorems which are direct consequences of Theorem 25.

Theorem 26. Let $G \in \mathcal{G}_{n}^{s c}$. If $\frac{\operatorname{det} W}{2^{\lfloor n / 2\rfloor}}$ is norm-free in $\mathbb{Z}[i]$, then for any $U \in \mathscr{U}_{G}, \ell(U) \in$ $\{1,1+i\}$. 
Proof. We claim that $\operatorname{gcd}(\ell(U), \overline{\ell(U)})=\ell(U)$. Otherwise, $\ell(U)$ must have an odd prime factor $p$ such that $p$ and $\bar{p}$ are not associates. Note that the $n$-th invariant factor $d_{n}$ clearly divides $\operatorname{det} W$. By Lemma 23, we have $\ell(U) \mid d_{n}$ and hence $p \mid \operatorname{det} W$. Therefore $\operatorname{lcm}(p, \bar{p}) \mid \operatorname{det} W$ as det $W$ is real or pure imaginary, according to Theorem 7 . Since $p$ and $\bar{p}$ are not associates, we have $\operatorname{lcm}(p, \bar{p})=N(p)$. Now $N(p) \mid \operatorname{det} W$ and hence $N(p) \mid \frac{\operatorname{det} W}{2^{\lfloor n / 2\rfloor}}$ as $N(p)$ is odd. This contradicts the assumption of this theorem. Thus the claim follows and hence $\ell(U) \in\{1,1+i\}$ by Theorem 25 .

Theorem 27. Let $G \in \mathcal{G}_{n}^{s c}$. If $\frac{\operatorname{det} W}{2^{\lfloor n / 2\rfloor}}$ is square-free in $\mathbb{Z}[i]$, then for any $U \in \mathscr{U}_{G}^{s c}$, $\ell(U) \in\{1,1+i\}$.

Proof. By Theorem 13, for any $U \in \mathscr{U}_{G}^{s c}$, we have that $\ell(U)$ and $\overline{\ell(U)}$ are associates, i.e., $\operatorname{gcd}(\ell(U), \overline{\ell(U)})=\ell(U)$. The theorem follows by Theorem 25 .

Now we give a few remarks.

Remark 28. Note that the notion of norm-free is a natural generalization of that of square-free from $\mathbb{Z}$ to $\mathbb{Z}[i]$, and therefore, Conjecture 24 is a natural generalization of Theorem 1 from ordinary graphs to self-conversed mixed graphs.

Remark 29. Theorem 26 is essentially the best possible, in the sense that if $\frac{\operatorname{det} W}{2^{\lfloor n / 2\rfloor}}$ is not norm-free for some Gaussian prime $p$, then Theorem 26 may not be true. This can be illustrated by the graph $G$ in Fig. 1. A direct calculation shows that $\operatorname{det} W=-68$, i.e.,

$$
\frac{\operatorname{det} W}{2^{\lfloor/ 2\rfloor}}=-17=-(4+i)(4-i)=i(4+i)(1+4 i),
$$

which is not norm-free since $\frac{\operatorname{det} W}{2^{5 / 2\rfloor}}$ has a pair of prime factors $4+i$ and $4-i$. Note that $G$ is not SHDGS, as the graph $H$ in Fig. 1 is cospectral with $G$ w.r.t. the generalized spectrum but is not isomorphic to $G$.

Remark 30. Let us consider the graph $G$ in Fig. 1 again. Note that $\frac{\operatorname{det} W}{2^{[5 / 2\rfloor}}$ is square-free in $\mathbb{Z}[i]$. The SNF of $W$ is $\operatorname{diag}(1,1,1,2,34)$. Let $U \in \mathscr{U}_{G}$. Then by Lemma 23 , we have $\ell(U) \mid 34$. Interestingly, many factors of 34 can never become the value of $\ell(U)$. Indeed, since $G$ satisfies the condition of Theorem 25 , we must have $\operatorname{gcd}(\ell(U), \overline{\ell(U)}) \in\{1,1+i\}$. It follows that $\ell(U) \in\{1,1+i, 4+i, 1+4 i, 3+5 i, 5+3 i\}$. Moreover, if we restrict $U$ in $\mathscr{U}_{G}^{s c}$, then Theorem 27 implies that $\ell(U) \in\{1,1+i\}$. Compared with the exhausting searching results explained in Example 14 earlier, the main gap of our theoretical results obtained here is that we cannot eliminate the possibility of $1+i$ as a divisor of $\ell(U)$.

Next, we return to Theorem 25. We first show that any odd prime $p$ cannot be a factor of $\operatorname{gcd}(\ell(U), \overline{\ell(U)})$ for any $U \in \mathscr{U}_{G}$. This fact is summarized as Theorem 31 in a more general form, which is the main focus of Section 4.1. In Section 4.2, we proceed to show that $\operatorname{gcd}(\ell(U), \overline{\ell(U)})$ has at most a single even prime factor.

\subsection{The case $p$ is odd}

Theorem 31. Let $G \in \mathcal{G}_{n}^{s c}$ such that $\operatorname{det} W \neq 0$. Let $U \in \mathscr{U}_{G}$ with level $\ell$. For any odd Gaussian prime $p$, if $p^{2} \nmid \operatorname{det} W$, then $p \nmid \operatorname{gcd}(\ell, \bar{\ell})$. 
Lemma 32. Let $G \in \mathcal{G}_{n}^{\text {sc }}$ such that $\operatorname{det} W \neq 0$. Let $U \in \mathscr{U}_{G}$ with level $\ell$. Let $p \in \Gamma$ be any odd prime factor of $d_{n}$, where $d_{n}$ is the $n$-th invariant factor of the SNF of $W$. If $p \mid \operatorname{gcd}(\ell, \bar{\ell})$ and $\operatorname{rank}_{p} W=n-1$, then there exists a Gaussian integral vector $z_{0}$ with $z_{0} \not \equiv 0(\bmod p), z_{0} \not \equiv 0(\bmod \bar{p})$ and a Gaussian integer $\lambda_{0}$ such that

$$
\begin{gathered}
z_{0}^{*} A^{k} z_{0} \equiv 0 \quad(\bmod N(\operatorname{lcm}(p, \bar{p}))), \text { for any } k \geqslant 0, \\
W^{*} z_{0} \equiv 0 \quad(\bmod \operatorname{lcm}(p, \bar{p})),
\end{gathered}
$$

and

$$
A z_{0} \equiv \lambda_{0} z_{0} \quad(\bmod \operatorname{lcm}(p, \bar{p})) .
$$

In particular, $z_{0}^{*} z_{0} \equiv 0(\bmod N(\operatorname{lcm}(p, \bar{p})))$ and $e^{*} z_{0} \equiv 0(\bmod \operatorname{lcm}(p, \bar{p}))$.

Proof. Let $H \in \mathcal{G}_{n}$ such that $\mathscr{U}_{G}=\{U\}$. Write $U_{1}=\ell U$. We consider the following two cases:

Case 1: $p$ and $\bar{p}$ are associates.

In this case, $\operatorname{lcm}(p, \bar{p})=p$. By the definition of $\ell, U_{1}$ is Gaussian integral and $U_{1}$ contains a column $z_{0}$ such that $z_{0} \not \equiv 0(\bmod p)$. Since $U^{*} A^{k}(G) U=A^{k}(H)$ for any $k \geqslant 0$, we have $U_{1}^{*} A^{k}(G) U_{1}=\bar{\ell} \ell A^{k}(H) \equiv 0(\bmod N(\ell))$, which implies $U_{1}^{*} A^{k}(G) U_{1} \equiv 0$ $(\bmod N(p))$ since $N(p) \mid N(\ell)$. Therefore, $z_{0}^{*} A^{k}(G) z_{0} \equiv 0(\bmod N(p))$. As $W^{*}(G) U_{1}=$ $\ell W^{*}(H)$, we have $W^{*}(G) z_{0} \equiv 0(\bmod \ell)$ and hence $W^{*}(G) z_{0} \equiv 0(\bmod p)$.

By Lemma 22, we have $\operatorname{rank}_{p} W^{*}(G)=\operatorname{rank}_{p} W(G)$ and hence $\operatorname{rank}_{p} W^{*}(G)=n-1$. As $W^{*}(G) U_{1} \equiv 0(\bmod p)$ and $U_{1}$ contains a column $z_{0} \not \equiv 0(\bmod p)$, we see that $\operatorname{rank}_{p} U_{1}=1$ and hence there exists a Gaussian integral row vector $\gamma$ such that $U_{1} \equiv z_{0} \gamma(\bmod p)$. Suppose that $z_{0}$ is the $t$-th column of $U_{1}$. As $A(G) U_{1}=U_{1} A(H)$, we have

$$
A(G) z_{0} \equiv z_{0}\left(\gamma A_{t}(H)\right) \equiv \lambda_{0} z_{0} \quad(\bmod p),
$$

where $A_{t}(H)$ is the $t$-th column of $A(H)$ and $\lambda_{0}=\gamma A_{t}(H)$ is a Gaussian integer.

Case 2: $p$ and $\bar{p}$ are not associates.

In this case, $\operatorname{lcm}(p, \bar{p})=p \bar{p}=N(p)$. As $p \mid \operatorname{gcd}(\ell, \bar{\ell})$, we have $\bar{p} \mid \operatorname{gcd}(\ell, \bar{\ell})$ and hence $N(p) \mid \operatorname{gcd}(\ell, \bar{\ell})$, which clearly implies that $N(p) \mid \ell$. Note that $W^{*}(G) U_{1}=\ell W^{*}(H)$ and $W^{*}(G)=S \Lambda T$. We have $S \Lambda T U_{1} \equiv 0(\bmod N(p))$, which can be simplified to

$$
\Lambda T U_{1} \equiv 0 \quad(\bmod N(p))
$$

since $S$ is unimodular.

Write $\tilde{U}=T U_{1}$ and $e_{n}=(0, \ldots, 0,1)^{\mathrm{T}}$, an $n$-dimensional coordinate vector. As $\operatorname{rank}_{p} W(G)=n-1$, we must have that $p \nmid d_{i}$ for any $i \in\{1,2, \ldots, n-1\}$. Also $\bar{p} \nmid d_{i}$ for any $i \in\{1,2, \ldots, n-1\}$ as $d_{i}$ and $\overline{d_{i}}$ are associates. It follows from (28) that

$$
\tilde{U} \equiv\left(m_{1} e_{n}, m_{2} e_{n}, \ldots, m_{n} e_{n}\right) \quad(\bmod N(p))
$$

for some Gaussian integers $m_{1}, m_{2}, \ldots, m_{n}$. Write $u=T^{-1} e_{n}$. Then we have

$$
U_{1} \equiv\left(m_{1} u, m_{2} u, \ldots, m_{n} u\right) \quad(\bmod N(p)) .
$$


If $p \mid m_{i}$ for each $i \in\{1,2, \ldots, n\}$ then $\frac{U_{1}}{p}$ is Gaussian integral, contradicting the minimality of $\ell$. Thus, $p \nmid m_{i}$ for some $i \in\{1,2, \ldots, n\}$. Similarly, $\bar{p} \nmid m_{j}$ for some $j \in\{1,2, \ldots, n\}$. Also $u \not \equiv 0(\bmod p)$ and $u \not \equiv 0(\bmod \bar{p})$. Denote $c=\operatorname{gcd}\left(m_{1}, m_{2}, \ldots, m_{n}\right)$. Then $p \nmid c$ and $\bar{p} \nmid c$. Since the ring of Gaussian integers is Euclidian, there exist $n$ Gaussian integers $q_{1}, q_{2}, \ldots, q_{n}$ such that $c=q_{1} m_{1}+q_{2} m_{2}+\cdots+q_{n} m_{n}$. Write $q=\left(q_{1}, q_{2}, \ldots, q_{n}\right)^{\mathrm{T}}$ and let $z_{0}=U_{1} q$.

From (30), we have

$$
z_{0} \equiv\left(m_{1} u, m_{2} u, \ldots, m_{n} u\right) q \equiv c u \quad(\bmod N(p)) .
$$

Therefore, $z_{0} \not \equiv 0(\bmod p)$ and $z_{0} \not \equiv 0(\bmod \bar{p})$. Note that

$$
z_{0}^{*} A^{k}(G) z_{0}=\left(U_{1} q\right)^{*} A^{k}(G)\left(U_{1} q\right)=q^{*} U_{1}^{*} A^{k}(G) U_{1} q=l \bar{l} q^{*} U^{*} A^{k}(G) U q=l \bar{l} q^{*} A^{k}(H) q .
$$

We have $z_{0}^{*} A^{k}(G) z_{0} \equiv 0\left(\bmod N^{2}(p)\right)$ as $N(p) \mid \ell$. As $W^{*}(G) z_{0}=W^{*}(G) U_{1} q=$ $\ell W^{*}(G) U q=\ell W^{*}(H) q$, we have $W^{*}(G) z_{0} \equiv 0(\bmod N(p))$.

Let $\eta=\left(\frac{m_{1}}{c}, \frac{m_{1}}{c}, \ldots, \frac{m_{n}}{c}\right)$. It follows from $(30)$ and $(31)$ that $U_{1} \equiv z_{0} \eta(\bmod N(p))$. Now the same argument as in Case 1 shows that $A(G) z_{0} \equiv \lambda_{0} z_{0}(\bmod N(p))$ for some Gaussian integer $\lambda_{0}$. This finally completes the proof.

Lemma 33. Using the notations of Lemma 32, $\operatorname{Im}\left(\lambda_{0}\right) \equiv 0(\bmod \operatorname{lcm}(p, \bar{p}))$.

Proof. We first show $\operatorname{Im}\left(\lambda_{0}\right) \equiv 0(\bmod p)$. From $(26)$, we have

$$
A z_{0} \equiv \lambda_{0} z_{0} \quad(\bmod p) \quad \text { and } A z_{0} \equiv \lambda_{0} z_{0} \quad(\bmod \bar{p})
$$

Taking conjugation on both sides of the second congruence equation in (32), we have

$$
\bar{A} \overline{z_{0}} \equiv \overline{\lambda_{0}} \overline{z_{0}} \quad(\bmod p) .
$$

Since $G$ is self-converse, there exists a permutation matrix $P$ such that $\bar{A}=P^{-1} A P$. Thus, by $(33), P^{-1} A P \overline{z_{0}} \equiv \overline{\lambda_{0}} \overline{z_{0}}(\bmod p)$ and hence $A P \overline{z_{0}} \equiv \overline{\lambda_{0}} P \overline{z_{0}}(\bmod p)$. Write $z_{1}=P \overline{z_{0}}$. Suppose to the contrary that $\operatorname{Im}\left(\lambda_{0}\right) \not \equiv 0(\bmod p)$. Then $\lambda_{0} \not \equiv \overline{\lambda_{0}}(\bmod p)$ as $p$ is odd. As $z_{0}$ and $z_{1}$ are eigenvectors corresponding to different eigenvalues, they are linearly independent over $\mathbb{Z}[i] /(p)$. Moreover, $e^{*} z_{1}=e^{*} P \overline{z_{0}}=e^{*} \overline{z_{0}}=\overline{e^{*} z_{0}} \equiv 0(\bmod p)$ and hence $e^{*} A^{k} z_{1} \equiv\left(\overline{\lambda_{0}}\right)^{k} e^{*} z_{1} \equiv 0(\bmod p)$. Therefore, $W^{*} z_{1} \equiv 0(\bmod p)$. As $W^{*} z_{0} \equiv 0(\bmod p)$ and $z_{0}, z_{1}$ are linearly independent, we have $\operatorname{rank}_{p} W^{*} \leqslant n-2$, i.e., $\operatorname{rank}_{p} W \leqslant n-2$ by Lemma 22. This contradicts our assumption that $p^{2} \nmid \operatorname{det} W$.

Note that the above argument also holds if we interchange $p$ and $\bar{p}$. Thus we also have $\operatorname{Im}\left(\lambda_{0}\right) \equiv 0(\bmod \bar{p})$ and hence $\operatorname{Im}\left(\lambda_{0}\right) \equiv 0(\bmod \operatorname{lcm}(p, \bar{p}))$.

Lemma 34. If $\left(A-\lambda_{0} I\right) y \equiv s p^{j} z_{0}\left(\bmod p^{j+1}\right)$ for some $s \in \mathbb{Z}[i]$ and $j \in\{0\} \cup \mathbb{Z}^{+}$then

$$
W^{*} y \equiv e^{*} y\left(1, \lambda_{0}, \ldots, \lambda_{0}^{n-1}\right)^{\mathrm{T}} \quad\left(\bmod p^{j+1}\right) .
$$


Proof. We claim that $e^{*} A^{k} y \equiv \lambda_{0}^{k} e^{*} y\left(\bmod p^{j+1}\right)$ for $k=0,1, \ldots, n-1$. The case $k=0$ is trivial. Let $k<n-1$. Suppose that the claim holds for $k$ and we are going to check it for $k+1$. By Lemma $32, W^{*} z_{0} \equiv 0(\bmod p)$, and hence $e^{*} A^{k} z_{0} \equiv 0(\bmod p)$ as $e^{*} A^{k}$ is the $(k+1)$-th row of $W^{*}$. Thus, $e^{*} A^{k} s p^{j} z_{0} \equiv 0\left(\bmod p^{j+1}\right)$. Now, by the condition of this lemma and induction hypothesis,

$$
e^{*} A^{k+1} y \equiv e^{*} A^{k}\left(s p^{j} z_{0}+\lambda_{0} y\right) \equiv \lambda_{0} e^{*} A^{k} y \equiv \lambda_{0}^{k+1} e^{*} y \quad\left(\bmod p^{j+1}\right) .
$$

This proves the claim and the lemma follows.

Lemma 35. Using the notations of Lemma 32,

$$
z_{0}^{*}\left(A-\lambda_{0} I, z_{0}\right) \equiv 0 \quad(\bmod p) \quad \text { and } \operatorname{rank}_{p}\left(A-\lambda_{0} I, z_{0}\right)=n-1 .
$$

Proof. We claim that

$$
\operatorname{rank}_{p}\left(A-\lambda_{0} I\right) \geqslant n-2 .
$$

Suppose to the contrary that $\operatorname{rank}_{p}\left(A-\lambda_{0} I\right) \leqslant n-3$. Consider the equation $\left(A-\lambda_{0} I\right) z \equiv 0$ $(\bmod p)$ which has a nontrivial solution $z_{0}$. There are at least two nontrivial solutions $y_{1}$ and $y_{2}$ such that $z_{0}, y_{1}, y_{2}$ are linear independent over $\mathbb{Z}[i] /(p)$. If either of $y_{1}$ and $y_{2}$, say $y_{1}$, satisfies $e^{*} y_{1} \equiv 0(\bmod p)$, then it follows from Lemma 34 for $s=0$ that $z_{0}$ and $y_{1}$ are two linear independent solutions of $W^{*} z \equiv 0(\bmod p)$, which contradicts the fact that $\operatorname{rank}_{p} W^{*}=n-1$. Thus, $e^{*} y_{1} \not \equiv 0(\bmod p)$ and $e^{*} y_{2} \not \equiv 0(\bmod p)$. Let $y_{3}=\left(e^{*} y_{1}\right) y_{2}-\left(e^{*} y_{2}\right) y_{1}$. As $y_{1}$ and $y_{2}$ are linear independent, $y_{3} \neq \equiv 0(\bmod p)$. Note that $e^{*} y_{3}=0$. Thus, $W^{*} y_{3} \equiv 0(\bmod p)$. Therefore, $W^{*} z \equiv 0(\bmod p)$ has solutions $z_{0}$ and $y_{3}$, which are clearly independent over $\mathbb{Z}[i] /(p)$. This contradiction completes the proof of (36).

Next we show (35). By Lemma 32, $\left(A-\lambda_{0} I\right) z_{0} \equiv 0(\bmod \bar{p})$. Taking conjugate transpose and noting that $A^{*}=A$ and $\operatorname{Im}\left(\lambda_{0}\right) \equiv 0(\bmod p)$, we have $z_{0}^{*}\left(A-\lambda_{0} I\right) \equiv 0$ $(\bmod p)$. Combining with the fact that $z_{0}^{*} z_{0} \equiv 0(\bmod p)$, we obtain $z_{0}^{*}\left(A-\lambda_{0} I, z_{0}\right) \equiv 0$ $(\bmod p)$. As $z_{0}^{*} \not \equiv 0(\bmod p)$, we have

$$
\operatorname{rank}_{p}\left(A-\lambda_{0} I, z_{0}\right) \leqslant n-1 .
$$

Suppose to the contrary that the equality in (37) does not hold. Then, by (36), $\operatorname{rank}_{p}\left(A-\lambda_{0} I\right)=n-2$ and $z_{0}$ can be written as a linear combination of the columns of $A-\lambda_{0} I$, say $z_{0} \equiv\left(A-\lambda_{0} I\right) z_{1}(\bmod p)$.

As $\operatorname{rank}_{p}\left(A-\lambda_{0} I\right)=n-2,\left(A-\lambda_{0} I\right) y \equiv 0(\bmod p)$ has two solutions $z_{2}$ and $z_{3}$ which are independent over $\mathbb{Z}[i] /(p)$. Since $\left(A-\lambda_{0} I\right) z_{1} \equiv z_{0} \not \equiv 0(\bmod p), z_{1}$ cannot be written as a linear combination of $z_{2}$ and $z_{3}$. This implies that $z_{1}, z_{2}, z_{3}$ are linearly independent. Consider the equation $e^{*}\left(k_{1} z_{1}+k_{2} z_{2}+k_{3} z_{3}\right) \equiv 0(\bmod p)$ with three unknowns $k_{1}, k_{2}, k_{3}$. Clearly, it has at least two independent solutions over $\mathbb{Z}[i] /(p)$. Let $\left(a_{1}, a_{2}, a_{3}\right)^{\mathrm{T}}$ and $\left(b_{1}, b_{2}, b_{3}\right)^{\mathrm{T}}$ be such two solutions and write $\alpha=a_{1} z_{1}+a_{2} z_{2}+a_{3} z_{3}$ and $\beta=b_{1} z_{1}+b_{2} z_{2}+b_{3} z_{3}$. It is easy to see that $\alpha$ and $\beta$ are linearly independent over $\mathbb{Z}[i] /(p)$. Note that $\left(A-\lambda_{0} I\right) \alpha \equiv$ $a_{1} z_{0}$ and $e^{*} \alpha \equiv 0(\bmod p)$. It follows from Lemma 34 that $W^{*} \alpha \equiv 0(\bmod p)$. Similarly, $W^{*} \beta \equiv 0(\bmod p)$. Thus, we have found two independent solutions of $W^{*} z \equiv 0(\bmod p)$. This contradicts the fact that $\operatorname{rank}_{p} W^{*}=n-1$ and hence completes the proof of this lemma. 
Lemma 36. Using the notations of Lemma 32, $W^{*} z \equiv 0\left(\bmod p^{2}\right)$ has a solution $z \not \equiv 0$ $(\bmod p)$.

Proof. Note that $p^{2} \mid N(\operatorname{lcm}(p, \bar{p}))$ always hold. By Lemma 32, we have $z_{0}^{*} A z_{0} \equiv 0$ $\left(\bmod p^{2}\right)$ and $z_{0}^{*} z_{0} \equiv 0\left(\bmod p^{2}\right)$. Consequently,

$$
z_{0}^{*}\left(A-\lambda_{0} I\right) z_{0} \equiv 0 \quad\left(\bmod p^{2}\right) .
$$

Note that $\left(A-\lambda_{0} I\right) z_{0} \equiv 0(\bmod p)$ by Lemma 32 . We can rewrite $(38)$ as

$$
z_{0}^{*} \frac{\left(A-\lambda_{0} I\right) z_{0}}{p} \equiv 0 \quad(\bmod p) .
$$

Note that $z_{0}^{*} \not \equiv 0(\bmod p)$. It follows from Lemma 35 that $\frac{\left(A-\lambda_{0} I\right) z_{0}}{p}$ can be expressed as a linear combination of the columns of $\left(A-\lambda_{0} I, z_{0}\right)$ over the field $\mathbb{Z}[i] /(p)$. Write

$$
\frac{\left(A-\lambda_{0} I\right) z_{0}}{p} \equiv\left(A-\lambda_{0} I\right) y+s z_{0} \quad(\bmod p) .
$$

Multiplying each side by $p$, we have $\left(A-\lambda_{0} I\right) z_{0} \equiv\left(A-\lambda_{0} I\right) p y+s p z_{0}\left(\bmod p^{2}\right)$, i.e.,

$$
\left(A-\lambda_{0} I\right)\left(z_{0}-p y\right) \equiv s p z_{0} \quad\left(\bmod p^{2}\right) .
$$

By Lemma 34, we have

$$
W^{*}\left(z_{0}-p y\right) \equiv e^{*}\left(z_{0}-p y\right)\left(1, \lambda_{0}, \lambda_{0}^{2}, \ldots, \lambda_{0}^{n-1}\right)^{\mathrm{T}} \quad\left(\bmod p^{2}\right) .
$$

Claim: There exists a vector $z_{1}$ such that $e^{*} z_{1} \not \equiv 0(\bmod p)$ and $\left(A-\lambda_{0} I\right) z_{1} \equiv s z_{0}$ $(\bmod p)$ for some $s \in\{0,1\}$.

By Lemma $35, \operatorname{rank}_{p}\left(A-\lambda_{0} I\right)=n-2$ or $n-1$. We prove the claim by considering the following two cases:

Case 1: $\operatorname{rank}_{p}\left(A-\lambda_{0} I\right)=n-2$.

By the condition of this case, the subspace of the eigenvectors of $A$ corresponding to $\lambda_{0}$ is 2 -dimensional. Thus, there exists a vector $z_{1}$ with $\left(A-\lambda_{0} I\right) z_{1} \equiv 0(\bmod p)$ that is linearly independent with $z_{0}$. If $e^{*} z_{1} \equiv 0(\bmod p)$ then $W^{*} z \equiv 0(\bmod p)$ by Lemma 34 . Note that $W^{*} z_{0} \equiv 0(\bmod p)$. Thus, $\operatorname{rank}_{p} W^{*} \leqslant n-2$, a contradiction.

Case 2: $\operatorname{rank}_{p}\left(A-\lambda_{0} I\right)=n-1$.

Since $\operatorname{rank}_{p}\left(A-\lambda_{0} I, z_{0}\right)=n-1$, the condition of this case implies that $z_{0}$ can be expressed as a linear combination of the columns of $A-\lambda_{0} I$, i.e., there exists a vector $z_{1}$ such that $\left(A-\lambda_{0} I\right) z_{1} \equiv z_{0}(\bmod p)$. Since $z_{0} \not \equiv 0(\bmod p), z_{1} \not \equiv 0(\bmod p)$ and $z_{1}$ is not an eigenvector of $A$ corresponding to $\lambda$ (over $\mathbb{Z}[i] /(p)$ ). Thus, $z_{1}$ and $z_{0}$ are linearly independent. Finally, we also have $e^{*} z_{1} \not \equiv 0(\bmod p)$ by the same argument as in Case 1. This proves the claim.

Note that $e^{*}\left(z_{0}-p y\right) \equiv e^{*} z_{0} \equiv 0(\bmod p)$. By the claim, there exists a Gaussian integer $g$ such that $\frac{e^{*}\left(z_{0}-p y\right)}{p} \equiv g e^{*} z_{1}(\bmod p)$, i.e,

$$
e^{*}\left(z_{0}-p y\right) \equiv g p e^{*} z_{1} \quad\left(\bmod p^{2}\right)
$$


By Lemma 34, we have $W^{*} z_{1} \equiv e^{*} z_{1}\left(1, \lambda_{0}, \ldots, \lambda_{0}^{n-1}\right)^{\mathrm{T}}(\bmod p)$ and hence

$$
g p W^{*} z_{1} \equiv g p e^{*} z_{1}\left(1, \lambda_{0}, \ldots, \lambda_{0}^{n-1}\right)^{\mathrm{T}} \quad\left(\bmod p^{2}\right) .
$$

It follows from $(42),(43)$ and $(44)$ that $W^{*}\left(z_{0}-p y\right) \equiv g p W^{*} z_{1}\left(\bmod p^{2}\right)$, i.e., $W^{*}\left(z_{0}-p y-\right.$ $\left.g p z_{1}\right) \equiv 0\left(\bmod p^{2}\right)$. This completes the proof of this lemma as $z_{0}-p y-g p z_{1} \equiv z_{0} \not \equiv 0$ $(\bmod p)$.

Proof of Theorem 31. We may assume $p \in \Gamma$. Suppose to the contrary that $p \mid \operatorname{gcd}(\ell, \bar{\ell})$. Then by Lemma $23, \ell \mid d_{n}$ and hence $p \mid d_{n}$. Note that $\operatorname{det} W=u d_{1} d_{2} \cdots d_{n}$ for some unit $u$. As $p^{2} \nmid \operatorname{det} W$, we find that $p \nmid d_{k}$ for any $k \in\{1,2, \ldots, n-1\}$ and $p^{2} \nmid d_{n}$. Thus, $\operatorname{rank}_{p} W=n-1$. It follows from Lemmas 32 and 36 that $W^{*} z \equiv 0\left(\bmod p^{2}\right)$ has a solution $z \not \equiv 0(\bmod p)$. Using Lemma $21, p^{2} \mid d_{n}$. This is a contradiction and hence completes the proof.

\subsection{The case $p=1+i$}

Theorem 37. Let $G \in \mathcal{G}_{n}^{\text {sc }}$ such that $\operatorname{det} W(G) \neq 0$. Let $U \in \mathscr{U}_{G}$ with level $\ell$. If $2^{\left\lfloor\frac{n}{2}\right\rfloor+1} \nmid \operatorname{det} W$, then $2 \nmid \ell$.

Set $k=\left\lfloor\frac{n}{2}\right\rfloor$. Let $\tilde{W}$ and $\tilde{W}_{1}$ be the matrices defined as follows:

$$
\tilde{W}= \begin{cases}\left(e, A e, \ldots, A^{k-1} e\right) & \text { if } n \text { is even } \\ \left(A e, A^{2} e, \ldots, A^{k} e\right) & \text { if } n \text { is odd }\end{cases}
$$

and

$$
\tilde{W}_{1}= \begin{cases}\left(e, A^{2} e, \ldots, A^{2 k-2} e\right) & \text { if } n \text { is even } \\ \left(A^{2} e, A^{4} e, \ldots, A^{2 k} e\right) & \text { if } n \text { is odd }\end{cases}
$$

Lemma 38. Let $G \in \mathcal{G}_{n}^{s c}$ such that $\frac{\operatorname{det} W}{2^{\left\lfloor\frac{n}{2}\right\rfloor}}$ is odd. Then the columns of $\tilde{W}$ constitute a set of fundamental solutions to $W^{*} z \equiv 0(\bmod 1+i)$.

Proof. We only consider the case that $n$ is even while the odd case can be proved in a similar way. Note that $e^{*} e=n \equiv 0(\bmod 2)$. It follows from Lemma 15 that $W^{*} \tilde{W} \equiv 0$ $(\bmod 2)$ and hence $W^{*} \tilde{W} \equiv 0(\bmod 1+i)$. Thus, each column of $\tilde{W}$ is a solution to $W^{*} z \equiv 0(\bmod 1+i)$. By Corollary $19, \operatorname{rank}_{1+i} W=\left\lceil\frac{n}{2}\right\rceil$ and hence any set of fundamental solutions has exactly $n-\left\lceil\frac{n}{2}\right\rceil=k$ vectors. Note that $\tilde{W}$ has exactly $k$ columns. By Lemma 17 , these $k$ columns are linearly independent and hence constitute a set of fundamental solutions.

By Lemma 15 and the fact that $e^{*} e \equiv 0(\bmod 2)$ when $n$ is even, one easily sees that all entries in $W^{*} \tilde{W}_{1}$ are divisible by 2 . That is, $\frac{W^{*} \tilde{W}_{1}}{2}$ is Gaussian integral. We show that this matrix has full column rank over $\mathbb{Z}[i] /(1+i)$, which is a generalization of $[17$, Lemma 3.10] for undirected graphs. The previous proof can be extended easily to mixed graphs.

Lemma 39. Let $G \in \mathcal{G}_{n}^{s c}$ such that $\frac{\operatorname{det} W}{2^{\left\lfloor\frac{n}{2}\right\rfloor}}$ is odd. Then we have $\operatorname{rank}_{1+i} \frac{W^{*} \tilde{W}_{1}}{2}=\left\lfloor\frac{n}{2}\right\rfloor$. 
Proof. We consider the following two cases:

Case 1: $n$ is even.

By Theorem 7, we have $\operatorname{det} W=u 2^{n / 2} b$, where $u$ is a unit and $b$ is an odd integer. Thus, $\operatorname{det} W^{*} W=2^{n} b^{2}$ and hence $\operatorname{det} \frac{W^{*} W}{2}=b^{2}$. Therefore, $\operatorname{rank}_{1+i} \frac{W^{*} W}{2}=n$. Thus, the $n$ columns of $\frac{W^{*} W}{2}$ are linearly independent, which clearly implies that $\frac{W^{*} \tilde{W}_{1}}{2}$ are also linearly independent. Thus $\operatorname{rank}_{1+i} \frac{W^{*} \tilde{W}_{1}}{2}=\left\lfloor\frac{n}{2}\right\rfloor$.

Case 2: $n$ is odd.

Let $W^{\prime}$ be the matrix obtained from $W$ by doubling the first column. Then $\frac{W^{*} W^{\prime}}{2}$ is Gaussian integral. As $\operatorname{det} W=u 2^{(n-1) / 2} b$ (b is odd), we have $\operatorname{det} W^{*} W^{\prime}=2^{n} b^{2}$ and hence $\frac{W^{*} W^{\prime}}{2}$ has full rank $n$. Thus, $\frac{W^{*} \tilde{W}_{1}}{2}$ must have full column rank, i.e, $\operatorname{rank}_{1+i} \frac{W^{*} \tilde{W}_{1}}{2}=$ $\left\lfloor\frac{n}{2}\right\rfloor$.

Lemma 40. Let $G \in \mathcal{G}_{n}^{\text {sc }}$ such that $\frac{\operatorname{det} W}{2^{\left\lfloor\frac{n}{2}\right\rfloor}}$ is odd. Let $U \in \mathscr{U}_{G}$ has level $\ell$. If $2 \mid \ell$ then there exists a Gaussian integral $n$-dimensional vector $u \neq \equiv 0(\bmod 1+i)$ and $a\left\lfloor\frac{n}{2}\right\rfloor$-dimensional vector $x \not \equiv 0(\bmod 1+i)$ such that $u^{*} A^{k} u \equiv 0(\bmod 4), W^{*} u \equiv 0(\bmod 2)$ and $\tilde{W} x \equiv u$ $(\bmod 2)$.

Proof. Let $H \in \mathcal{G}_{n}^{s c}$ such that $\mathscr{U}_{G}(H)=\{U\}$. Write $U_{1}=\ell U$. Note that $1+i$ divides $\ell$. Due to the minimality of $N(\ell), U_{1}$ contains a column $u$ such that $u \neq \equiv 0(\bmod 1+i)$. Since $U_{1}^{*} A^{k}(G) U_{1}=\bar{\ell} \ell U^{*} A^{k}(G) U=\bar{\ell} \ell A^{k}(H)$ and $2 \mid \ell$, we have $U_{1}^{*} A^{k} U_{1} \equiv 0(\bmod 4)$ and hence $u^{*} A^{k} u \equiv 0(\bmod 4)$. Similarly, we have $W^{*}(G) U_{1}=\ell W^{*}(G) U=\ell W^{*}(H) \equiv 0$ $(\bmod 2)$ and hence $W^{*} u \equiv 0(\bmod 2)$. It remains to find a vector $x \not \equiv 0(\bmod 1+i)$ such that $\tilde{W} x \equiv u(\bmod 2)$.

Since $W^{*} u \equiv 0(\bmod 2)$, we have $W^{*} u \equiv 0(\bmod 1+i)$. It follows from Lemma 38 that $u$ can be expressed as a linear combination of columns of $\tilde{W}$, that is, there exists a vector $v$ such that $u \equiv \tilde{W} v(\bmod 1+i)$.

Claim: $\tilde{W} y \equiv \frac{u-\tilde{W} v}{1+i}(\bmod 1+i)$ has a solution for unknown vector $y$.

Write $b=\frac{u-\tilde{W} v}{1+i}$. Let $z$ be any vector satisfying $\tilde{W}^{*} z \equiv 0(\bmod 1+i)$. If we can show that $b^{*} z \equiv 0(\bmod 1+i)$ always holds, then the equations $\tilde{W}^{*} x \equiv 0(\bmod 1+i)$ and $(\tilde{W}, b)^{*} x \equiv 0(\bmod 1+i)$ have the same solutions, which implies that $\operatorname{rank}_{1+i} \tilde{W}^{*}=$ $\operatorname{rank}_{1+i}(\tilde{W}, b)^{*}$, i.e., $\operatorname{rank}_{1+i} \tilde{W}=\operatorname{rank}_{1+i}(\tilde{W}, b)$ and hence the claim follows.

As $\tilde{W}^{*}$ has full column rank $k=\left\lfloor\frac{n}{2}\right\rfloor$, the solution space of $\tilde{W}^{*} x \equiv 0(\bmod 1+i)$ has dimension $\left\lceil\frac{n}{2}\right\rceil$. As $\tilde{W}^{*} W \equiv 0(\bmod 2)$, we have $\tilde{W}^{*} W \equiv 0(\bmod 1+i)$. By Corollary 19 we have $\operatorname{rank}_{1+i} W=\left\lceil\frac{n}{2}\right\rceil$ and hence $z$ belongs to the column space of $W$. Thus, we can write $z \equiv W a(\bmod 1+i)$ for some vector $a$. Therefore,

$$
\begin{aligned}
z^{*} b & \equiv(W a)^{*} \frac{u-\tilde{W} v}{1+i} \\
& \equiv a^{*} \frac{W^{*} u-W^{*} \tilde{W} v}{1+i} \\
& \equiv 0 \quad(\bmod 1+i)
\end{aligned}
$$

where the last congruence holds because $W^{*} u \equiv 0(\bmod 2)$ and $W^{*} \tilde{W} \equiv 0(\bmod 2)$. Thus, $b^{*} z \equiv 0(\bmod 1+i)$ and the Claim holds. 
Let $x=v+(1+i) y$. By the claim, we have $\tilde{W}(1+i) y \equiv u-\tilde{W} v(\bmod 2)$. Thus, $\tilde{W} x \equiv u(\bmod 2)$. Finally, as $u \not \equiv 0(\bmod 1+i)$, we must have $x \not \equiv 0(\bmod 1+i)$. This completes the proof of this lemma.

Proof of Theorem 3\%. Suppose to the contrary that $2 \mid \ell$. Let $u$ and $x$ be vectors as described in Lemma 40 . As $\tilde{W} x \equiv u(\bmod 2)$, we have $u=\tilde{W} x+2 \beta$ for some vector $\beta$. It follows that

$$
\begin{aligned}
u^{*} A^{j} u & =(\tilde{W} x+2 \beta)^{*} A^{j}(\tilde{W} x+2 \beta) \\
& =x^{*} \tilde{W}^{*} A^{j} \tilde{W} x+2 \beta^{*} A^{j} \tilde{W} x+2 x^{*} \tilde{W}^{*} A^{j} \beta+4 \beta^{*} A^{j} \beta \\
& =x^{*} \tilde{W}^{*} A^{j} \tilde{W} x+4 \operatorname{Re}\left(\beta^{*} A^{j} \tilde{W} x\right)+4 \beta^{*} A^{j} \beta \\
& \equiv x^{*} \tilde{W}^{*} A^{j} \tilde{W} x \quad(\bmod 4) .
\end{aligned}
$$

Since $u^{*} A^{j} u \equiv 0(\bmod 4)$ by Lemma 40, from (50), we have

$$
x^{*} \tilde{W}^{*} A^{j} \tilde{W} x \equiv 0 \quad(\bmod 4) \quad \text { for } j=0,1, \ldots, n-1 .
$$

Define

$$
\left(j_{1}, j_{2}, \ldots, j_{k}\right)= \begin{cases}\left(0,1, \ldots, \frac{n}{2}-1\right) & \text { if } n \text { is even } \\ \left(1,2, \ldots, \frac{n-1}{2}\right) & \text { if } n \text { is odd }\end{cases}
$$

where $k=\left\lfloor\frac{n}{2}\right\rfloor$. Then $\tilde{W}=\left[A^{j_{1}} e, A^{j_{2}} e, \ldots, A^{j_{k}} e\right]$. Let $R^{(l)}=\left(r_{s, t}^{(l)}\right)=\tilde{W}^{*} A^{l} \tilde{W}, l=$ $0,1, \ldots, n-1$. Then we have

$$
r_{s, t}^{(l)}=e^{*} A^{j_{s}+j_{t}+l} e .
$$

We claim that $R^{(l)}$ is a real symmetric matrix with each entry even. Clearly, by (53), $r_{s, t}^{(l)}$ is real and $r_{s, t}^{(l)}=r_{t, s}^{(l)}$. It remains to show that $2 \mid r_{s, t}^{(l)}$. If $j_{s}+j_{t}+l>0$ then the claim follows by Lemma 15. Now assume $j_{s}=j_{t}=l=0$. According to (52), $n$ must be even in this case. Thus, $e^{*} A^{j_{s}+j_{t}+l} e$ is an even integer and the claim also holds. This proves the claim. It follows that

$$
\begin{aligned}
x^{*} \tilde{W}^{*} A^{l} \tilde{W} x & =\sum_{1 \leqslant s, t \leqslant k} x_{s}^{*} r_{s, t}^{(l)} x_{t} \\
& =\sum_{1 \leqslant s \leqslant k} x_{s}^{*} r_{s, s}^{(l)} x_{s}+\sum_{1 \leqslant s<t \leqslant k} x_{s}^{*} r_{s, t}^{(l)} x_{t}+\sum_{1 \leqslant t<s \leqslant k} x_{s}^{*} r_{s, t}^{(l)} x_{t} \\
& =\sum_{1 \leqslant s \leqslant k} x_{s}^{*} r_{s, s}^{(l)} x_{s}+\sum_{1 \leqslant s<t \leqslant k}\left(x_{s}^{*} r_{s, t}^{(l)} x_{t}+x_{t}^{*} r_{t, s}^{(l)} x_{s}\right) \\
& =\sum_{1 \leqslant s \leqslant k} x_{s}^{*} r_{s, s}^{(l)} x_{s}+\sum_{1 \leqslant s<t \leqslant k} r_{s, t}^{(l)}\left(x_{s}^{*} x_{t}+\overline{x_{s}^{*} x_{t}}\right) \\
& =\sum_{1 \leqslant s \leqslant k} x_{s}^{*} r_{s, s}^{(l)} x_{s}+\sum_{1 \leqslant s<t \leqslant k} 2 r_{s, t}^{(l)} \operatorname{Re}\left(x_{s}^{*} x_{t}\right) \\
& \equiv \sum_{1 \leqslant s \leqslant k} x_{s}^{*} r_{s, s}^{(l)} x_{s} \quad(\bmod 4) .
\end{aligned}
$$


Thus, from (51), we have

$$
\sum_{1 \leqslant s \leqslant k} x_{s}^{*} r_{s, s}^{(l)} x_{s} \equiv 0 \quad(\bmod 4)
$$

Note that $x_{s}^{*} x_{s}$ and $x_{s}$ have the same Gaussian parity, i.e., $x_{s}^{*} x_{s} \equiv x_{s}(\bmod 1+i)$. As $r_{s, s}^{(l)}$ is an even integer, we have $x_{s}^{*} r_{s, s}^{(l)} x_{s} \equiv r_{s, s}^{(l)} x_{s}(\bmod 2(1+i))$. Thus, from $(54)$, we have

$$
\left[r_{1,1}^{(l)}, r_{2,2}^{(l)}, \ldots, r_{k, k}^{(l)}\right] x \equiv 0 \quad(\bmod 2(1+i)), l=0,1, \ldots, n-1 .
$$

Moreover, we have

$$
\begin{aligned}
& \left(\begin{array}{cccc}
r_{1,1}^{(0)} & r_{2,2}^{(0)} & \ldots & r_{k, k}^{(0)} \\
r_{1,1}^{(1)} & r_{2,2}^{(1)} & \ldots & r_{k, k}^{(1)} \\
\cdots & \cdots & \cdots & \cdots \\
r_{1,1}^{(n-1)} & r_{2,2}^{(n-1)} & \cdots & r_{k, k}^{(n-1)}
\end{array}\right) \\
= & \left(\begin{array}{cccc}
e^{*} A^{2 j_{1}} e & e^{*} A^{2 j_{2}} e & \cdots & e^{*} A^{2 j_{k}} e \\
e^{*} A^{2 j_{1}+1} e & e^{*} A^{2 j_{2}+1} e & \cdots & e^{*} A^{2 j_{k}+1} e \\
\vdots & \vdots & \cdots & \vdots \\
e^{*} A^{2 j_{1}+n-1} e & e^{*} A^{2 j_{2}+n-1} e & \cdots & e^{*} A^{2 j_{k}+n-1} e
\end{array}\right) \\
= & \left(\begin{array}{c}
e^{*} \\
e^{*} A \\
\vdots \\
e^{*} A^{n-1}
\end{array}\right)\left(A^{2 j_{1}} e, A^{2 j_{2}} e, \ldots, A^{2 j_{k}} e\right) \\
= & W^{*} \tilde{W}_{1} .
\end{aligned}
$$

Thus, we can rewrite (55) as

$$
W^{*} \tilde{W}_{1} x \equiv 0 \quad(\bmod 2(1+i)) .
$$

As $\frac{W^{*} \tilde{W}_{1}}{2}$ is Gaussian integral, the equation is equivalent to

$$
\frac{W^{*} \tilde{W}_{1}}{2} x \equiv 0 \quad(\bmod 1+i)
$$

From Lemma 39, we know that $\operatorname{rank}_{1+i} \frac{\tilde{W}^{*} \tilde{W}_{1}}{2}=\left\lfloor\frac{n}{2}\right\rfloor$. Thus, $x \equiv 0(\bmod 1+i)$. This contradiction completes the proof of Theorem 37.

Proof of Theorem 25. Theorem 25 follows immediately from Theorems 31 and 37.

\section{Two extremal cases}

We shall verify Conjecture 8 for two extremal cases that $G$ is either an undirected graph (no edge is oriented) or a self-converse oriented graph (each edge is oriented). 


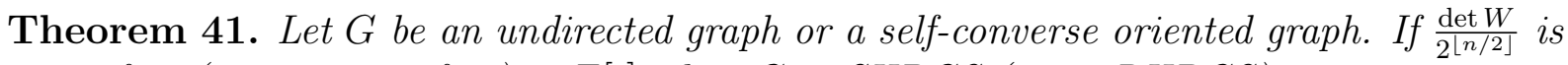
norm-free (resp. square-free) in $\mathbb{Z}[i]$, then $G$ is SHDGS (resp. RHDGS).

Note that the undirected case of Theorem 41 strengthens Theorem 1 . That is, even in $\mathcal{G}_{n}^{s c}$, which includes all undirected graphs on $[n]$ as a proper subset, the only graphs $\mathbb{R}$-cospectral to $G$ are isomorphic to $G$ if $\frac{\operatorname{det} W}{2^{\lfloor n / 2\rfloor}}$ is square-free in $\mathbb{Z}[i]$. Moreover, if $\frac{\operatorname{det} W}{2^{\lfloor n / 2\rfloor}}$ is norm-free, then $G$ is determined uniquely by generalized Hermitian spectrum even among all mixed graphs.

For a positive integer $n$, let $\mathcal{M}_{n}$ denote the set of all $n \times n$ Hermitian Gaussian integral matrices with vanishing diagonal entries. For $k \geqslant 1$ and $s \geqslant 0$, let

$$
U_{k, s}=\left(\begin{array}{ccccc}
U_{0} & & & & \\
& U_{0} & & & \\
& & \ddots & & \\
& & & U_{0} & \\
& & & & I_{s}
\end{array}\right)
$$

be a matrix of order $2 k+s$, where $U_{0}=\frac{1}{1+i}\left(\begin{array}{ll}1 & i \\ i & 1\end{array}\right)$ and $I_{s}$ is the identity matrix of order $s$. It is easy to see that $U^{*} U=I, U e=e$ and $\ell\left(U_{k, s}\right)=1+i$.

Lemma 42. Let $U$ be an $n \times n$ Gaussian rational unitary matrix with $U e=e$ and $\ell(U)=$ $1+i$. Then there exist two permutation matrices $P$ and $Q$ such that $P U Q=U_{k, n-2 k}$ for some $k \geqslant 1$.

Proof. We prove the lemma by induction on $n$. Since $\ell(U)=1+i$ and $U e=e, U$ has a row which contains at least two nonzero entries. Let $\tilde{U}=(1+i) U$ and $P_{1}, Q_{1}$ be two permutation matrices such that the first two entries of the first row in $P_{1} \tilde{U} Q_{1}$ are non-zero. Let $\left(a_{1}, a_{2}, \ldots, a_{n}\right)$ denote the first row of $P_{1} \tilde{U} Q_{1}$. Now we have $a_{1}+a_{2}+\cdots+a_{n}=1+i$ and $\left|a_{1}\right|^{2}+\left|a_{2}\right|^{2}+\cdots+\left|a_{n}\right|^{2}=2$. As each $a_{j}$ is Gaussian integral and $a_{1}, a_{2} \neq 0$, one must have $\left(a_{1}, a_{2}, \ldots, a_{n}\right)=(1, i, 0, \ldots, 0)$ or $(i, 1,0, \ldots, 0)$. We may assume $\left(a_{1}, a_{2}, \ldots, a_{n}\right)=$ $(1, i, 0, \ldots, 0)$ since otherwise we can interchange the first two columns of $Q_{1}$. Let $\alpha$ and $\beta$ denote the first and second columns of $P_{1} \tilde{U} Q_{1}$. Note that $U^{\mathrm{T}} e=e$. Similar considerations indicate that both $\alpha$ and $\beta$ have exactly two non-zero entries ( 1 and $i$ ), thus $\alpha=(1,0, \ldots, 0, i, 0, \ldots, 0)$ and $\beta=(i, 0, \ldots, 0,1,0, \ldots, 0)$. As $\alpha^{*} \beta=0$ the position of $i$ in $\alpha$ agrees with the position of 1 in $\beta$. Thus, there exists a permutation matrix $P_{2}$ such that $P_{2} P_{1} \tilde{U} Q_{1}$ has the following form:

$$
P_{2} P_{1} \tilde{U} Q_{1}=\left(\begin{array}{ccccc}
1 & i & 0 & \cdots & 0 \\
i & 1 & 0 & \cdots & 0 \\
0 & 0 & * & \cdots & * \\
\vdots & \vdots & \vdots & \cdots & \vdots \\
0 & 0 & * & \cdots & *
\end{array}\right) .
$$


Equivalently,

$$
P_{2} P_{1} U Q_{1}=\left(\begin{array}{ll}
U_{0} & \\
& U_{1}
\end{array}\right)
$$

for some $U_{1}$ of order $n-2$.

If $n=2$ we are done. Suppose that $n \geqslant 3$. Let $e_{1}$ denote the all-one vector of dimension $n-2$. It is easy to see that $U_{1}$ is a Gaussian rational unitary matrix with $U_{1} e_{1}=e_{1}$. Moreover, $\ell\left(U_{1}\right) \mid \ell(U)$, that is $\ell\left(U_{1}\right) \in\{1,1+i\}$. If $\ell\left(U_{1}\right)=1$ then $U_{1}$ is a permutation matrix. Let

$$
Q_{2}=\left(\begin{array}{cc}
I_{2} & \\
& U_{1}^{-1}
\end{array}\right)
$$

Then $Q_{2}$ is a permutation matrix and $P_{2} P_{1} U Q_{1} Q_{2}=U_{1, n-2}$. This proves the lemma for the case that $\ell\left(U_{1}\right)=1$. If $\ell\left(U_{1}\right)=1+i$, by induction hypothesis, there exist permutation matrices $P^{\prime}$ and $Q^{\prime}$ such that $P^{\prime} U_{1} Q^{\prime}=U_{k^{\prime}, n-2-2 k^{\prime}}$. Let

$$
P_{3}=\left(\begin{array}{cc}
I_{2} & \\
& P^{\prime}
\end{array}\right) \text { and } Q_{3}=\left(\begin{array}{cc}
I_{2} & \\
& Q^{\prime}
\end{array}\right) .
$$

Then $P_{3} P_{2} P_{1} U Q_{1} Q_{3}=U_{1+k^{\prime}, n-2-2 k^{\prime}}$. This completes the proof of this lemma.

Lemma 43. Let $A$ be a (0,1)-matrix or a $(0, \pm i)$-matrix of order $n$ and $B=U_{k, s}^{*} A U_{k, s}$ where $2 k+s=n$. If each entry of $B$ belongs to $\{0,1, i,-i\}$, then $A=B$.

Proof. Write

$$
A=\left(\begin{array}{ccccc}
A_{1,1} & A_{1,2} & \cdots & A_{1, k} & A_{1, k+1} \\
A_{2,1} & A_{2,2} & \cdots & A_{2, k} & A_{2, k+1} \\
\cdots & \cdots & \cdots & \cdots & \cdots \\
A_{k, 1} & A_{k, 2} & \cdots & A_{k, k} & A_{k, k+1} \\
A_{k+1,1} & A_{k+1,2} & \cdots & A_{k+1, k} & A_{k+1, k+1}
\end{array}\right)
$$

where $A_{j, j}$ is a square matrix of order 2 for $j \in[k]$, and $A_{k+1, k+1}$ is of order $s$. We have

$$
U_{k, s}^{*} A U_{k, s}=\left(\begin{array}{ccccc}
U_{0}^{*} A_{1,1} U_{0} & U_{0}^{*} A_{1,2} U_{0} & \cdots & U_{0}^{*} A_{1, k} U_{0} & U_{0}^{*} A_{1, k+1} \\
U_{0}^{*} A_{2,1} U_{0} & U_{0}^{*} A_{2,2} U_{0} & \cdots & U_{0}^{*} A_{2, k} U_{0} & U_{0}^{*} A_{2, k+1} \\
\cdots & \cdots & \cdots & \cdots & \cdots \\
U_{0}^{*} A_{k, 1} U_{0} & U_{0}^{*} A_{k, 2} U_{0} & \cdots & U_{0}^{*} A_{k, k} U_{0} & U_{0}^{*} A_{k, k+1} \\
A_{k+1,1} U_{0} & A_{k+1,2} U_{0} & \cdots & A_{k+1, k} U_{0} & A_{k+1, k+1}
\end{array}\right) .
$$

Let $\Omega_{1}$ (resp. $\left.\Omega_{2}\right)$ denote the set of all $(0,1)$-matrices (resp. $(0, \pm i)$-matrices) $C$ of order 2 such that each entry of $U_{0}^{*} C U_{0}$ belongs to $\{0,1, i,-i\}$. Direct calculation shows that

$$
\begin{gathered}
\Omega_{1}=\left\{\left(\begin{array}{ll}
0 & 0 \\
0 & 0
\end{array}\right),\left(\begin{array}{ll}
1 & 0 \\
0 & 1
\end{array}\right),\left(\begin{array}{ll}
0 & 1 \\
1 & 0
\end{array}\right),\left(\begin{array}{ll}
1 & 1 \\
1 & 1
\end{array}\right)\right\}, \\
\Omega_{2}=\left\{\left(\begin{array}{ll}
0 & 0 \\
0 & 0
\end{array}\right), \pm\left(\begin{array}{ll}
i & 0 \\
0 & i
\end{array}\right), \pm\left(\begin{array}{ll}
0 & i \\
i & 0
\end{array}\right), \pm\left(\begin{array}{ll}
i & i \\
i & i
\end{array}\right), \pm\left(\begin{array}{cc}
-i & i \\
i & -i
\end{array}\right)\right\},
\end{gathered}
$$


and moreover, $U_{0}^{*} C U_{0}=C$ for each $C \in \Omega_{k}, k=1,2$. This proves that $U_{0}^{*} A_{i, j} U_{0}=A_{i, j}$ for $i, j \in[k]$. Similar argument shows that each column (resp. row) of $A_{i, k+1}$ (resp. $\left.A_{k+1, i}\right)$ is of form $u(1,1, \ldots, 1)^{\mathrm{T}}$ (resp. $\left.u(1,1, \ldots, 1)\right)$, where $u \in\{0,1\}$ when $A$ is a $(0-1)$ matrix, and $u \in\{0, i,-i\}$ when $A$ is a $(0, \pm i)$-matrix. Therefore, $U_{0}^{*} A_{i, k+1}=A_{i, k+1}$ and $A_{k+1, i} U_{0}=A_{k+1, i}$. This completes the proof.

Proof of Theorem 41. We only prove the strong determination part of this theorem using Theorem 26. The restrictive determination part can be settled in exactly the same way using Theorem 27. Suppose to the contrary that $G$ is not SHDGS. Then there exists a $U$ such that $\ell(U) \neq 1$, and $\{U\}=\mathscr{U}_{G}(H)$, i.e., $U^{*} A(G) U=A(H)$ for some $H \in \mathcal{G}_{n}$. By Theorem $27, \ell(U) \in\{1,1+i\}$ and we must have $\ell(U)=1+i$.

By Lemma 42, there exist two permutation matrices $P$ and $Q$ such that $P U Q=$ $U_{k, n-2 k}$, i.e., $U=P^{*} U_{k, n-2 k} Q^{*}$ for some $k \geqslant 1$. Therefore, we have

$$
\left(P^{*} U_{k, n-2 k} Q^{*}\right)^{*} A(G) P^{*} U_{k, n-2 k} Q^{*}=A(H) .
$$

Write $A_{1}=P A(G) P^{*}, B_{1}=Q^{*} A(H) Q$ and let $G_{1}, H_{1}$ be two graphs with adjacency matrices $A_{1}$ and $B_{1}$ respectively. Now (64) is equivalent to

$$
U_{k, n-2 k}^{*} A_{1} U_{k, n-2 k}=B_{1} .
$$

It follows from Lemma 43 that $A_{1}=B_{1}$, i.e., $G_{1}=H_{1}$. Now, from (65), we have

$$
U_{k, n-2 k}^{*} W\left(G_{1}\right)=W\left(H_{1}\right)=W\left(G_{1}\right) .
$$

As $W\left(G_{1}\right)=P W(G)$ and $\operatorname{det} W(G) \neq 0$, we have $\operatorname{det} W\left(G_{1}\right) \neq 0$, that is $W\left(G_{1}\right)$ is invertible. Note that $U_{k, n-2 k}^{*}$ is not the identity matrix. This contradicts (66) and hence completes the proof.

Example 44. Let $\vec{P}_{4}$ be the directed path of order 4 whose Hermitian adjacency matrix and walk-matrix are given as follows, respectively,

$$
A=\left(\begin{array}{cccc}
0 & i & 0 & 0 \\
-i & 0 & i & 0 \\
0 & -i & 0 & i \\
0 & 0 & -i & 0
\end{array}\right) \quad \text { and } W=\left(\begin{array}{cccc}
1 & i & 0 & i \\
1 & 0 & 1 & i \\
1 & 0 & 1 & -i \\
1 & -i & 0 & -i
\end{array}\right)
$$

We have $\operatorname{det} W=-4$. Note that $\frac{\operatorname{det} W}{2^{\lfloor/ 2\rfloor}}$ equals -1 and hence is trivially norm-free. Using Theorem 41, the graph $\vec{P}_{4}$ is SHDGS.

Indeed, we can show that all directed paths of even order are SHDGS by using Theorem 41, based on the calculation of det $W$. The proof is given in the Appendix. 


\section{Concluding remarks}

In this paper, we are mainly concerned with the generalized spectral characterizations of mixed graphs. We propose two related notions: strong determination (SHDGS) and restrictive determination (RHDGS) by generalized Hermitian spectrum. When $G$ is an ordinary graph, both notions strengthen the usual determination by generalized spectrum.

Given a self-converse mixed graph $G$ of order $n$ such that $\frac{\operatorname{det} W}{2^{\left\lfloor\frac{n}{2}\right\rfloor}}$ (which is always a real or pure imaginary integer) is norm-free in $\mathbb{Z}[i]$, we show that for any mixed graph $H$ that is $\mathbb{R}$-cospectral to $G$, there exists a Gaussian rational unitary matrix $U$ with $U e=e$ such that $U^{*} A(G) U=A(H)$ and $(1+i) U$ is a Gaussian integral matrix. Such a unitary matrix $U$ is very close to a permutation matrix, and therefore gives strong evidences for the conjecture that all self-converse mixed graphs satisfying the above condition are SHDGS. For restrictive determination, similar results are also obtained under a weaker condition that $\frac{\operatorname{det} W}{2^{\left\lfloor\frac{n}{2}\right\rfloor}}$ is square-free in $\mathbb{Z}[i]$. We have verified our conjecture in two extremal cases when $G$ has only directed edges or only undirected edges. In particular, the undirected case strengthens a recent result of the first author [17], and as a consequence of the directed case, we show that every directed path of even order is SHDGS.

However, regarding Conjecture 24, new insights and techniques are still needed to eliminate the possibility that $\ell(U)=1+i$. We leave it as an interesting and challenging future work.

\section{Acknowledgements}

We would like to thank Haiying Shan for his help on programming in SageMath.

\section{References}

[1] S. Akbari, A. Ghafaria, M. Nahvib, M. A. Nematollahia, Mixed paths and cycles determined by their spectrum, Linear Algebra Appl., 586 (2020) 325-346.

[2] B. Mohar, Hermitian adjacency spectrum and switching equivalence of mixed graphs, Linear Algebra Appl., 489 (2016) 324-340.

[3] E. R. van Dam, W. H. Haemers, Which graphs are determined by their spectrum? Linear Algebra Appl., 373 (2003) 241-272.

[4] E. R. van Dam, W. H. Haemers, Developments on spectral characterizations of graphs, Discrete Math., 309 (2009) 576-586.

[5] K. Guo, B. Mohar, Hermitian adjacency matrix of digraphs and mixed graphs, $J$. Graph Theory, 85(1) (2017) 324-340.

[6] C. R. Johnson, M. Newman, A note on cospectral graphs, J. Combin. Theory, Ser. B, 28 (1980) 96-103.

[7] J. Liu, X. Li, Hermitian-adjacency matrices and Hermitian energies of mixed graphs, Linear Algebra Appl., 466 (2015) 182-207. 
[8] L. Mao, F. Liu, W. Wang, A new method for constructing graphs determined by their generalized spectrum, Linear Algebra Appl., 477(15) (2015) 112-127.

[9] B. D. Makay, A. Piperno, Practical graph isomorphism, II, J. Symb. Comput., 60 (2014) 94-112.

[10] https://mathoverflow.net/questions/338274/an-equality-about-sin-function. [Accessed on 7th March, 2020.]

[11] L. Qiu, Y. Ji, W. Wang, A new arithmetic criterion for graphs being determined by their generalized Q-spectrum, Discrete Math., 342 (2019) 2770-2782.

[12] L. Qiu, Y. Ji, W. Wang, On the generalized spectral characterizations of Eulerian graphs, Electron. J. Combin., 26(1) (2019) \#P9.

[13] SageMath, the Sage Mathematics Software System (Version 8.9), The Sage Developers, 2019, https://www. sagemath.org.

[14] K. Tran, Discriminants of polynomials related to Chebyshev polynomials: The "Mutt and Jeff" syndrome. J. Math. Anal. Appl., 383(1)(2011) 120-129.

[15] W. Wang, Generalized spectral characterization revisited, Electron. J. Combin., 20(4) (2013) \#P4.

[16] W. Wang, On the spectral characterization of graphs, PhD Thesis, Xi'an Jiaotong University, 2006 (in Chinese).

[17] W. Wang, A simple arithmetic criterion for graphs being determined by their generalized spectra, J. Combin. Theory, Ser. B, 122 (2017) 438-451.

[18] W. Wang, C.-X. Xu, An excluding algorithm for testing whether a family of graphs are determined by their generalized spectra, Linear Algebra Appl., 418 (2006) 62-74.

[19] W. Wang, C.-X. Xu, A sufficient condition for a family of graphs being determined by their generalized spectra, European J. Combin., 27 (2006) 826-840.

[20] P. Wissing, E.R. van Dam, The negative tetrahedron and the first infinite family of connected digraphs that are strongly determined by the Hermitian spectrum, $J$. Combin. Theory, Ser. A, 173 (2020) 105232.

\section{Appendix}

Theorem A1. Each directed path $\vec{P}_{2 m}$ is SHDGS for any $m \geqslant 1$.

We need the following equality, which was first established in [8] for ordinary graphs.

Lemma A2. Let $G$ be a mixed graph and $\lambda_{1}, \lambda_{2}, \ldots, \lambda_{n}$ be $n$ eigenvalues of $A(G)$. If $\xi_{1}, \xi_{2}, \ldots, \xi_{n}$ are $n$ normalized eigenvectors of $A(G)$ corresponding to $\lambda_{1}, \lambda_{2}, \ldots, \lambda_{n}$, respectively. Then we have

$$
\operatorname{det} W^{*}=u \prod_{1 \leqslant k<j \leqslant n}\left(\lambda_{j}-\lambda_{k}\right) \prod_{j=1}^{n} e^{*} \xi_{j}
$$

for some unit $u$ in $\mathbb{Z}[i]$. 
Proof. Write $A=A(G)$. For $s \in[n-1]$, we have

$$
\begin{aligned}
e^{*} A^{s}\left(\xi_{1}, \xi_{2}, \ldots, \xi_{n}\right) & =\left(\lambda_{1}^{s} e^{*} \xi_{1}, \lambda_{2}^{s} e^{*} \xi_{2}, \ldots, \lambda_{n}^{s} e^{*} \xi_{n}\right) \\
& =\left(\lambda_{1}^{s}, \lambda_{2}^{s}, \ldots, \lambda_{n}^{s}\right)\left(\begin{array}{llll}
e^{*} \xi_{1} & & & \\
& e^{*} \xi_{2} & & \\
& & \ddots & \\
& & & e^{*} \xi_{n}
\end{array}\right)
\end{aligned}
$$

and hence

$$
\begin{aligned}
W^{*}\left(\xi_{1}, \xi_{2} \ldots, \xi_{n}\right) & =\left(\begin{array}{c}
e^{*} \\
e^{*} A \\
\vdots \\
e^{*} A^{n-1}
\end{array}\right)\left(\xi_{1}, \xi_{2}, \ldots, \xi_{n}\right) \\
& =\left(\begin{array}{cccc}
1 & 1 & \ldots & 1 \\
\lambda_{1} & \lambda_{2} & \ldots & \lambda_{n} \\
\ldots & \ldots & \ldots & \ldots \\
\lambda_{1}^{n-1} & \lambda_{2}^{n-1} & \ldots & \lambda_{n}^{n-1}
\end{array}\right)\left(\begin{array}{cccc}
e^{*} \xi_{1} & & & \\
& e^{*} \xi_{2} & & \\
& & \ddots & \\
& & & e^{*} \xi_{n}
\end{array}\right)
\end{aligned}
$$

Therefore, $\operatorname{det} W^{*} \operatorname{det}\left(\xi_{1}, \xi_{2}, \ldots, \xi_{n}\right)=\prod_{1 \leqslant k<j \leqslant n}\left(\lambda_{j}-\lambda_{k}\right) \prod_{j=1}^{n} e^{*} \xi_{j}$.

Note that $\left(\xi_{1}, \xi_{2}, \ldots, \xi_{n}\right)$ is unitary and hence its determinant is a unit in $Z[i]$. This proves the lemma.

The discriminant of a polynomial $P(x)$ of degree $n$ and leading coefficient $a_{n}$ is

$$
\operatorname{Disc}_{x} P(x)=a_{n}^{2 n-2} \prod_{1 \leqslant k<j \leqslant n}\left(r_{j}-r_{k}\right)^{2} .
$$

where $r_{1}, r_{2}, \ldots, r_{n}$ are the roots of $P(x)$. Let $U_{n}(x)$ be the Chebyshev polynomial of the second kind. The discriminant of $U_{n}(x)$ has an elegant formula:

$$
\operatorname{Disc}_{x} U_{n}(x)=2^{n^{2}}(n+1)^{n-2} ;
$$

see [14] for a proof.

\section{Lemma A3.}

$$
\prod_{1 \leqslant k<j \leqslant n}\left(2 \cos \frac{\pi}{n+1} j-2 \cos \frac{\pi}{n+1} k\right)= \pm 2^{\frac{n}{2}}(n+1)^{\frac{n}{2}-1} .
$$

Proof. The leading coefficient of $U_{n}(x)$ is $2^{n}$ and the roots are $\cos \frac{\pi}{n+1} j, j=1,2, \ldots, n$. Thus, by (A.1) and (A.2), we have

$$
2^{n(2 n-2)} \prod_{1 \leqslant k<j \leqslant n}\left(\cos \frac{\pi}{n+1} j-\cos \frac{\pi}{n+1} k\right)^{2}=2^{n^{2}}(n+1)^{n-2},
$$

which implies (A.3). 


\section{Lemma A4.}

$$
\sum_{k=1}^{n} \sin ^{2} \frac{k \pi}{n+1} j=\frac{n+1}{2}, \text { for } j=1,2, \ldots, n .
$$

Proof. Write $\alpha=\frac{\pi}{n+1} j$. Note that $\sin \alpha \neq 0$ as $0<\alpha<\pi$. We have

$$
\begin{aligned}
\sum_{k=1}^{n} \sin ^{2} k \alpha & =\frac{n}{2}-\frac{1}{2} \sum_{k=1}^{n} \cos 2 k \alpha \\
& =\frac{n}{2}-\frac{1}{2 \sin \alpha} \sum_{k=1}^{n} \cos 2 k \alpha \sin \alpha \\
& =\frac{n}{2}-\frac{1}{2 \sin \alpha} \sum_{k=1}^{n} \frac{1}{2}(\sin (2 k+1) \alpha-\sin (2 k-1) \alpha) \\
& =\frac{n}{2}-\frac{1}{4 \sin \alpha}(\sin (2 n+1) \alpha-\sin \alpha) .
\end{aligned}
$$

As $(2 n+1) \alpha=(2 n+2) \alpha-\alpha=2 \pi j-\alpha$, we have $\sin (2 n+1) \alpha=-\sin \alpha$ and hence $\sum_{k=1}^{n} \sin ^{2} k \alpha=\frac{n+1}{2}$, as desired.

\section{Lemma A5.}

$$
\prod_{j=1}^{2 n} \sum_{k=1}^{2 n}(-i)^{k} \sin \frac{k \pi}{2 n+1} j=(-1)^{n} \frac{2 n+1}{2^{n}} .
$$

Proof. The proof of this lemma is due to Fedor Petrov [10]. We have

$$
\sum_{k=1}^{2 n}(-i)^{k} \sin \frac{k \pi}{2 n+1} j=\sum_{k=0}^{2 n}(-i)^{k} \sin \frac{k \pi}{2 n+1} j=\frac{h(j)-h(-j)}{2 i},
$$

where

$$
h(j)=\sum_{k=0}^{2 n} e^{i\left(-\pi / 2+\frac{\pi j}{2 n+1}\right) k}=\frac{1-e^{-i \pi(2 n+1) / 2+i \pi j}}{1-e^{i\left(-\pi / 2+\frac{\pi j}{2 n+1}\right)}}=\frac{1+i(-1)^{n+j}}{1+i e^{i \frac{\pi j}{2 n+1}}} .
$$

The numerators for $j$ and $-j$ are the same, and

$$
\frac{1}{1+i e^{i \theta}}-\frac{1}{1+i e^{-i \theta}}=\frac{1-i e^{i \theta}}{1+e^{2 i \theta}}-\frac{1-i e^{-i \theta}}{1+e^{-2 i \theta}}=\frac{1-i e^{2 i \theta}}{1+e^{2 i \theta}}
$$

so the left expression of (A.4) reads as

$$
(-1)^{n} 2^{-2 n} \prod_{j=1}^{2 n}\left(1+i(-1)^{n+j}\right) \frac{1-e^{2 i \theta}}{1+e^{2 i \theta}}
$$

where $\theta=\frac{\pi j}{2 n+1}$. The product $\prod_{j=1}^{2 n}\left(1+i(-1)^{n+j}\right)$ equals $2^{n}$, since the product of two consecutive terms equals 2 . It remains to prove that

$$
\prod_{j=1}^{2 n} \frac{1-e^{\frac{2 \pi i}{2 n+1} j}}{1+e^{\frac{2 \pi i}{2 n+1} j}}=2 n+1 .
$$


Write $\omega=e^{2 \pi i /(2 n+1)}$ and $P(z)=\prod_{j=1}^{2 n}\left(z-\omega^{j}\right)$. As $1, \omega, \omega^{2}, \ldots, \omega^{2 n}$ are the roots of $z^{2 n+1}-1=0$, we have

$$
P(z)=\prod_{j=1}^{2 n}\left(z-\omega^{j}\right)=\frac{z^{2 n+1}-1}{z-1}=z^{2 n}+z^{2 n-1}+\cdots+1 .
$$

Therefore,

$$
\prod_{j=1}^{2 n} \frac{1-\omega^{j}}{1+\omega^{j}}=\prod_{j=1}^{2 n} \frac{1-\omega^{j}}{-1-\omega^{j}}=\frac{P(1)}{P(-1)}=2 n+1
$$

as desired.

Proof of Theorem A1. Write $n=2 m$. Let $A$ and $B$ be the Hermitian adjacency matrix of $\vec{P}_{n}$ and the ordinary adjacency matrix of its underlying graph $P_{n}$. That is,

$$
A=\left(\begin{array}{ccccc}
0 & i & & & \\
-i & 0 & i & & \\
& -i & \ddots & \ddots & \\
& & \ddots & 0 & i \\
& & & -i & 0
\end{array}\right), B=\left(\begin{array}{ccccc}
0 & 1 & & & \\
1 & 0 & 1 & & \\
& 1 & \ddots & \ddots & \\
& & \ddots & 0 & 1 \\
& & & 1 & 0
\end{array}\right)
$$

It is known that $A$ and $B$ have the same spectrum. Taking $Q=\operatorname{diag}\left(-i,(-i)^{2}, \ldots,(-i)^{n}\right)$, then we have $Q^{*}=\operatorname{diag}\left(i, i^{2}, \ldots, i^{n}\right)$ and

$$
A=Q B Q^{*}
$$

It is well-known that $\operatorname{det}(x I-B)$ is $U_{n}(x / 2)$, where $U_{n}(x)$ is the Chebyshev polynomial of the second kind. The spectra of $B$ are $\lambda_{j}=2 \cos \frac{\pi}{n+1} j(j=1,2, \ldots, n)$ and

$$
\xi_{j}=\left(\sin \frac{\pi}{n+1} j, \quad \sin \frac{2 \pi}{n+1} j, \ldots, \sin \frac{n \pi}{n+1} j\right)^{\mathrm{T}}
$$

is an eigenvector to $\lambda_{j}$. Let

$$
\eta_{j}=Q \xi_{j}=\left((-i) \sin \frac{\pi}{n+1} j,(-i)^{2} \sin \frac{2 \pi}{n+1} j, \ldots,(-i)^{n} \sin \frac{n \pi}{n+1} j\right)^{\mathrm{T}} .
$$

It follows from (A.6) that $\eta_{j}$ is an eigenvector of $B$ corresponding to the eigenvalue $\lambda_{j}$.

Note that $\frac{\eta_{j}}{\left\|\eta_{j}\right\|}$ is the normalized eigenvector, where $\left\|\eta_{j}\right\|=\sqrt{\eta_{j}^{*} \eta_{j}}$. It follows from Lemma A2 that

$$
\operatorname{det} W^{*}=u \prod_{1 \leqslant k<j \leqslant n}\left(\lambda_{j}-\lambda_{k}\right) \prod_{j=1}^{n} \frac{1}{\left\|\eta_{j}\right\|} \prod_{j=1}^{n} e^{*} \eta_{j}
$$


for some unit $u$ in $\mathbb{Z}[i]$. By Lemmas A3, A4 and A5, we have

$$
\left|\operatorname{det} W^{*}\right|=2^{\frac{n}{2}}(n+1)^{\frac{n}{2}-1} \cdot\left(\frac{1}{\sqrt{\frac{n+1}{2}}}\right)^{n} \cdot \frac{2 \cdot \frac{n}{2}+1}{2^{\frac{n}{2}}}=2^{\frac{n}{2}} .
$$

Note that $\vec{P}_{2 m}$ is self-converse. Theorem 7 implies that $\operatorname{det} W$ is real or pure imaginary. Thus, det $W=u 2^{\frac{n}{2}}$ for some unit $u$ in $\mathbb{Z}[i]$. This shows that $\vec{P}_{2 m}$ is SHDGS by Theorem 41. 